\title{
La regulación de las nanotecnologías en Argentina. Sobre polisemia e inestabilidad de los nano-objetos
}

\author{
Regulating Nanotechnologies in Argentina. \\ About polysemy and instability of nano-objects

\section{A regulação das nanotecnologias na Argentina. Sobre polissemia e instabilidade dos nano-objetos}

Resumen: Este trabajo se enmarca en una línea de los Estudios Sociales de la Ciencia y la Tecnología (ESCT) que estudia las relaciones entre derecho, democracia y nanotecnologías. Se analiza la experiencia de regulación de las nanotecnologías en Argentina en el periodo 2001 2017, a partir del rastreo y procesamiento de proyectos legislativos, iniciativas institucionales $y$ discursos expertos comprometidos en la coproducción de un saber científico/jurídico "nano". Se identificaron dos grandes etapas. En la primera etapa (2001-2006) se aborda el proceso que lleva a la creación por decreto de la Fundación Argentina de Nanotecnología (FAN) y se reseñan las críticas que recibió el modelo adoptado. En la segunda etapa (2007 en adelante), que inicia con la creación del Ministerio de Ciencia, Tecnología e Innovación Productiva (MINCyT), se estudia un modelo de gobierno "nano" que combina la institucionalidad experta y la estandarización normativa global. Se concluye que el carácter inestable y polisémico de los nanoobjetos posiciona distintos tipos de conocimiento como mediadores de los formatos regulatorios e institucionales. En este sentido, el trabajo pretende contribuir: a) en general, a las discusiones actuales sobre los modos que tiene el derecho de estabilizar objetos controvertidos; b) en particular, a describir cómo los operadores legales visualizan a los nano-objetos en Argentina.

Palabras clave: estudios sociales de la ciencia y la tecnología (ESCT), derecho y nanotecnologías, nanotecnologías en Argentina; nano-objetos, regulación de objetos controvertidos.

$\stackrel{\leftrightarrow}{\hookrightarrow}$ Conicet - Centro de Investigaciones en Derecho FCJS-UNL (Argentina). Becario Conicet (Argentina). Docente de Derecho Civil II (Obligaciones) y de Derecho de Daños en la Facultad de Ciencias Jurídicas y Sociales de la Universidad Nacional del Litoral (FCJSUNL). Investigador Centro de Investigaciones en Derecho (FCJS-UNL).

Orcid ID: https://orcid.org/0000-0002-7395-2630

$\bowtie$ gbailo@fcjs.unl.edu.ar

Revista de la Facultad de Derecho, (45), 2018, e20184501

DOI: $10.22187 / \mathrm{rfd} 2018 \mathrm{n} 45 \mathrm{a} 1$

ISSN 0797-8316 / eISSN 2301-0665

1 de 49 
Abstract: This paper is framed in the area of Science and Technology Studies (STS) that studies the relationships between law, democracy and nanotechnologies. It is analyzed nanotechnologies regulatory experience in Argentina between 2001-2017 by tracing and processing national bills, institutional initiatives and expert discourses committed on the coproduction of a "nano" scientific-legal knowledge. It is identified two major phases. The first phase (20012006) leads to the creation by decree of the Argentinian Nanotechnology Foundation (FAN) and includes several critics about it. The second phase (2007-present) begins with the creation of Science, Technology and Productive Innovation Ministry (MINCyT) and adopts a "nano" governance model that combines expert institutionalism with the implementation of global standardization regulation. It is concluded that the instability and polysemic character of nanoobjects repositions various types of knowledge as mediators of regulatory and institutional models. This paper aims to contribute: a) in general, to current discussions about the legal modes of stabilizing controversial objects; b) in particular, to describe how legal actors visualize nanoobjects in Argentina.

Keywords: science and technology studies (STS), law and nanotechnologies, nanotechnologies in Argentina, nano-objects, controversial objects regulation.

Resumo: Este trabalho faz parte da linha de Estudos Sociais da Ciência e Tecnologia (ESCT) que estuda as relações entre direito, democracia e nanotecnologias. Analisa-se a experiência de regulação das nanotecnologias na Argentina no período 2001-2017, a partir do rastreamento e processamento de projetos legislativos, iniciativas institucionais e discursos de especialistas comprometidos na coprodução dum conhecimento científico/jurídico “nano”. Identificaram-se duas grandes etapas. A primeira (2001-2006) aborda o processo que leva à criação por decreto da Fundação Argentina de Nanotecnologia (FAN) e inclui as críticas ao modelo adotado. A segunda (2007- presente), que começa com a criação do Ministério da Ciência, Tecnologia e Inovação Produtiva (MINCyT), adota um modelo de governo "nano" que combina institucionalidade especializada e estandardização normativa global. Conclui-se que a natureza instável e polissêmica dos nano-objetos posiciona diferentes tipos de conhecimento como mediadores dos formatos regulatórios e institucionais. Nesse sentido, o trabalho visa contribuir: a) em geral, às discussões atuais sobre as formas em que o direito estabiliza objetos polêmicos; b) em particular, a descrever como os operadores legais visualizam aos nano-objetos na Argentina.

Palavras-chave: estudos sociais da ciência e tecnologia (ESCT); direito e nanotecnologias, nanotecnologias na Argentina, nano-objetos, regulação de objetos polêmicos.

Recibido: 20180322

Aceptado: 20180425 


\section{Sumario}

Introducción. La inestabilidad de los nano-objetos. 1. Etapa 2001-2006. Puertas de entrada de los nano-objetos al mundo regulatorio. 2. Tensiones y renegociación de la nanografía. 3. Etapa 2007-actualidad. Los nano-objetos reordenando el espacio de lo público y lo privado. 4. Las experiencias regulatorias. 5. La polisemia de la nano-objetualidad. Algunas conclusiones provisorias

\section{Introducción. La inestabilidad de los nano-objetos}

Este trabajo se enmarca en una línea de los Estudios Sociales de la Ciencia y la Tecnología (ESCT) que estudia las relaciones entre derecho, democracia y nanotecnologías. Se analiza la experiencia de regulación de las nanociencias y nanotecnologías (N\&N) en Argentina en el período 20012017, a partir del rastreo y procesamiento de proyectos legislativos, iniciativas institucionales y discursos expertos comprometidos en la coproducción de un saber científico/jurídico "nano".

Se entiende que el estudio de la nano-objetualidad exige revisitar la relación que existe entre los distintos tipos de conocimientos comprometidos en la tarea de regulación y construcción de la institucionalidad pública, es decir, explorar la medida en que los modelos regulatorios dependen de los agenciamientos inscriptos en los objetos de la tecnociencia.

Una primera respuesta a ello, casi intuitiva, presupone que los nano-objetos son entidades perfectamente demarcadas, listas para ser reguladas por los medios que el derecho tiene a su disposición. En esta respuesta se asume que las sociedades esperan cosas diferentes de la ciencia y del derecho. La ciencia debe describir el universo "nano" tal cual se presenta, y el derecho debe custodiar esos avances regulando mejor las relaciones sociales que están bajo su cuidado. Este enfoque ha predominado en los textos que provienen de las instituciones tradicionales del Estado, v.g. en los proyectos legislativos 
presentados en el Congreso de la Nación en el período 2001-2014 y en los fundamentos del Decreto del Poder Ejecutivo Nacional No 380/2005 que creó la Fundación Argentina de Nanotecnología (FAN).

Una segunda respuesta concibe a los nano-objetos como objetos altamente inestables y controvertidos. En esta tesitura, la estabilización de la nano-objetualidad es una empresa común, que incumbe tanto a la ciencia como al derecho, y que moviliza una gran cantidad de recursos y crea sus propias audiencias. Si las controversias sobre estos procesos, que involucran a expertos, funcionarios políticos y a la ciudadanía en general, son parte de la política democrática moderna (cfr. Jasanoff, 2011, 20), el estudio de la estabilización y desestabilización de los nano-objetos impide aislarlos de sus redes institucionales y programas regulatorios (cfr. Laurent, 2017). El modelo de estabilización de los nano-objetos en Argentina ha sido asumido principalmente por expertos involucrados en redes de investigación de N\&N que fueron alcanzando distintos niveles de adscripción en la institucionalidad pública. Este modelo se reforzó a partir de la sincronización de la nano-objetualidad local con la estandarización técnica global y por la prácticamente nula participación ciudadana en los mecanismos de gobierno de las N\&N.

De esta manera, las controversias que se tejen alrededor de los objetos de la tecnociencia problematizan la confianza en el conocimiento científico como custodio del interés público (cfr. Nelkin, 1995, 450), y ponen de manifiesto la posibilidad de reescribir las reglas de la práctica política en momentos de cambio científico y tecnológico (Nelkin, 1987, 284; Jasanoff, 2011). Como lo expresa Hermitte, que los objetos de la ciencia y la técnica pasen "del estado de objetos aleatorios y separados al de objeto político central" (2007, 18 ), conduce a modificaciones institucionales que involucran a todos los poderes del Estado $(2007,41)$.

\section{Etapa 2001-2006. Puertas de entrada de los nano-objetos al mundo regulatorio}

En Argentina, el primer proyecto de ley que incluyó a las nanotecnologías como una línea prioritaria de investigación data de Abril de 2001 y tuvo al Senado como cámara de origen ${ }^{1}$. En este documento se preveía la creación de un "Plan Estratégico de Ciencia y Tecnología" en cabeza de la autoridad de aplicación de ciencia y tecnología y la presentación de un "Informe Bianual" 
respecto al estado de su ejecución. El proyecto incluía la nanotecnología como un área "considerada prioritaria por la comunidad internacional" y reservaba a la autoridad de aplicación en ciencia y tecnología "las acciones conducentes para avanzar en materia de investigación"2.

En el año 2003 la Secretaría de Ciencia y Tecnología (SECyT) definió a las N\&N como áreas de interés y vacancia en Argentina (Salvarezza, 2011) En el año 2004 se incluyó en forma explícita a las N\&N en el Programa de Áreas de Vacancias (PAV) ${ }^{4}$ y se convocó a la constitución de Redes a través de la Agencia Nacional de Promoción Científica y Tecnológica $(\mathrm{ANPCyT})^{5}$. En 2005 también se crea el Centro Argentino-Brasileño de Nanociencia y Nanotecnología (CABNN), destinado a la coordinación de acciones conjuntas con Brasil, como la formación de recursos humanos y la realización de conferencias (Observatorio CTS, 2011; Salvarezza, 2011).

En Marzo de 2005, el diputado Jorge R. Giorgetti (PJ) presentó el primer proyecto legislativo que trató específicamente sobre nanotecnologías ${ }^{6}$. El proyecto constaba de 5 artículos, en los que proponía la "Creación del Instituto Argentino de Nanotecnología” en el Ministerio de Economía, en coordinación con la Secretaría de Ciencia y Técnica de la Nación?

En los fundamentos, se establecía que era necesario un cambio en el perfil productivo del país, y que la nanotecnología implica "el nacimiento de un nuevo paradigma industrial. Es una nueva revolución industrial. Es después de la informática y la biotecnología la que mayores posibilidades y capacidades tiene para influir en la salud, la riqueza y la vida de nuestra gente" ${ }^{\text {". Los }}$ fundamentos se encargaron de manifestar algunas de sus aplicaciones en los sectores productivos, y aluden a la conveniencia que Argentina encuentre "su especialización" en los mercados 9 .

Sin embargo, la puerta de entrada de los nano-objetos al derecho vigente no fueron los proyectos legislativos precitados, sino una gestión del Poder Ejecutivo que culminó con el dictado del Decreto 380/2005. En los primeros días de Noviembre de 2004, los principales periódicos de Argentina se hacían eco de los anuncios del Ministro de Economía de la Nación, Roberto Lavagna, respecto a un plan para el desarrollo local de nanotecnologías. La iniciativa se sustentaba en un acuerdo con la empresa multinacional Lucent-Technologies (ex Bell Laboratories) para fabricar en el país semi- 
conductores y chips en miniatura ${ }^{10}$. Debido al interés en el desarrollo de piezas microscópicas con aplicaciones en medicina, óptica y comunicaciones, en este plan se preveía la participación de la Comisión Nacional de Energía Atómica (CNEA), la firma Invap de Río Negro, y se extendía la invitación a otras empresas que desearan formar parte de la iniciativa. El Ministerio justificaba la necesidad de buscar cooperación empresarial en el alto costo requerido para la producción nanotecnológica.

Las actuaciones administrativas en la órbita del Ministerio de Economía y Producción de la Nación -Exp-S01:0063812/2005- finalizaron el 27 de Abril de 2005 con el dictado del Decreto 380/2005. El decreto autorizó al Ministerio a constituir la Fundación Argentina de Nanotecnología (FAN), sobre la base de dos anexos que formaban parte de la norma: el proyecto de "Acta de Constitución" $" 11$ y el proyecto de "Estatuto"12.

En los fundamentos del decreto se mencionó la necesidad de "competir internacionalmente en la aplicación y desarrollo de micro y nanotecnologías que aumenten el valor agregado de productos destinados al consumo interno y la exportación", y se extendió el interés inicial centrado en el desarrollo de piezas microscópicas a otros sectores de la economía, "entre los que sobresalen el de los alimentos, y otros de base tecnológica, como el de la biotecnología"13.

Lavagna dejó el Ministerio en noviembre de 2005 y la FAN se puso en marcha bajo la gestión de su reemplazante, Felisa Miceli, quien desplazó la posición dominante de Lucent. En agosto de 2006, Miceli creó el Consejo Asesor de la FAN e hizo público que la fundación financiaría entre el 50 y el $80 \%$ del costo total de las iniciativas que se seleccionaran, con un monto máximo de dos millones de dólares (Iprofesional, 2006).

La fundamentación económica de los nano-objetos comenzó a ganar lugar en el discurso de los expertos que comenzaban a ocupar los nuevos espacios institucionales ${ }^{14}$. Lino Barañao, por ese entonces tesorero de la FAN y Director de la ANPCyT, definía a las nanotecnologías como "una nueva revolución industrial" que "va a impactar en toda la cadena productiva" (Iprofesional, 2006). En base a ello, Barañao entendía que la selección de proyectos requería de un criterio de gestión empresarial, que destinara el dinero a "las ideas con mayor impacto" (La Razón, 2006) ${ }^{15}$. 
Al mismo tiempo, el Plan Estratégico Nacional de Ciencia, Tecnología e Innovación "Bicentenario" (2006-2010) establecía -junto con otras áreas de estudio- a la Nanotecnología como área prioritaria. A partir de ello, la ANPCyT inició el Programa de Áreas Estratégicas (PAE), a través del cual se aprobaron dos proyectos relacionados con la Nanotecnología que permitieron conformar nuevas redes articuladas con actores del sector productivo (MINCyT, 2012b, 8).

\section{Tensiones y renegociación de la nanografía}

\section{Las tensiones}

La inscripción legal de los nano-objetos por la vía del Decreto 380/2005, aunque estilizada por la pluma economicista, no tardó en generar reacciones adversas en el campo político y científico.

En un Comunicado firmado por Dr. J. Raúl Grigera, la Comisión Directiva de la Asociación Física Argentina (AFA), hizo dos consideraciones respecto al Decreto 380/2005. En primer lugar, expresó su aprobación respecto de la inversión en el área, por ser "aquella en la que el mundo desarrollado está haciendo las más grandes inversiones previendo que el desarrollo futuro se sustentará sobre la nano y microtecnología”. En segundo lugar, que no resulta claro que en el proceso se hayan cumplido con los mecanismos previstos por los sistemas de acreditación y evaluación de proyectos con los que cuenta el sistema científico tecnológico nacional, regulados por la Ley $\mathrm{N}^{\mathrm{o}}$ $25.467^{16}$. La AFA cierra su Comunicado, diciendo que las instituciones que actualmente tienen involucrado personal e instalaciones en el desarrollo en la nano y micro-tecnología en nuestro país "deberían ver incrementadas sus capacidades evitando que se generen nuevas estructuras a costa de lo logrado hasta el presente".

En el campo parlamentario, la diputada Lilia Puig de Stubrin, que presidía la Comisión de Ciencia y Tecnología de la Cámara de Diputados, a través del proyecto de resolución 2844-D-05, solicitó al poder ejecutivo un informe sobre algunos puntos controvertidos del Decreto 380/2005 ${ }^{17}$. Se advertía respecto de la creación de la FAN por fuera del marco legal que regulaba las actividades de ciencia, tecnología e innovación productiva y sin la participación de la Secretaría de Ciencia y Tecnología. Señalaba que una ad- 
judicación directa de fondos terminaba quebrando el sistema científico-tecnológico nacional ${ }^{18}$.

La diputada también advertía que, según el estatuto, la FAN tiene márgenes para actividades más allá de la micro y nanotecnología, por lo que pidió también al Poder Ejecutivo que explique de quién serán las patentes de los productos que se desarrollen en la Fundación y "si se ha previsto que, por falta de empresas que desarrollen nanotecnología en el país, los recursos humanos que se capaciten podrían terminar en el exterior".

Por otra parte, el 30 de Septiembre de 2005, el Comité Nacional de Ética en la Ciencia y la Tecnología (CECTE $)^{19}$ emitió una declaración respecto del Decreto Nacional 380/2005. El Comité expresó que dicha normativa provocó inquietudes en los científicos del país, y en particular en la Asociación Física Argentina (ver ut supra), que solicitó una expedición del CECTE sobre los aspectos éticos comprometidos. El CECTE entendió que para concretar los resultados positivos de la inversión en Ciencia y Tecnología "se requiere que la gestión y el uso de fondos que son públicos (por lo tanto, aportados por la sociedad en su conjunto) se realice de modo totalmente transparente". A tales fines, prosigue el razonamiento, Argentina cuenta con la Ley 25.467, Ley Marco de Ciencia, Tecnología e Innovación, cuya finalidad es regular la investigación básica y tecnológica ${ }^{20}$.

Cabe destacar que el CECTE tiene entre sus objetivos el estudio y elaboración de protocolos sobre principios éticos en la práctica científica y tecnológica en relación a salud humana y medio ambiente. Sin embargo, el Comité en este informe apenas hizo mención, en una nota a pie de página, a que en materia de nanotecnologías "junto a los posibles beneficios existen potenciales consecuencias cuyas implicaciones sociales deben preverse y analizarse".

Paralelamente a estas discusiones, distintos documentos e investigaciones dieron cuenta de la influencia del gobierno estadounidense en la diagramación y financiación de proyectos en el sector nanotecnológico local (cfr. Ferrari, 2005a; Foladori, 2006; Andrini y Figueroa, 2008), lo que despertó críticas y resistencias en la comunidad científica (Página 12, 2005d) y en miembros del poder legislativo (Ferrari, 2005c), en especial frente a la posición esgrimida por el -por entonces- Secretario de Ciencia y Tecnología de la Nación (Ferrari, $2005 b)^{21}$. La controversia se originó a raíz de un pedido de informes -encabe- 
zado por la diputada Puig de Stubrin-en $2005^{22}$, a lo que siguió en 2006 el pedido de informes de otro grupo de diputados, pero esta vez en torno al financiamiento por parte de agencias militares estadounidenses de una Conferencia que se había realizado en Bariloche, lo cual desencadenó en la renuncia del gerente del Centro Atómico Bariloche (cfr. Foladori, 2006) ${ }^{23}$.

En 2007, el diario Página 12 -que se había abocado al seguimiento de varias controversias asociadas al gobierno de las N\&N en Argentina- recogió el testimonio de un especialista en biodiversidad que consideraba que la nanotecnología engloba dos mecanismos de regulación económica: los derechos de propiedad intelectual y el control tecnológico (Premici, 2007)24. El periódico también recoge las palabras de Daniel Lupi, por entonces director del Centro de Electrónica e Informática del INTI, que entendía que "el valor adicional de utilizar nanotecnología en un producto servirá para beneficiar, en las primeras etapas, a las empresas que ya fabrican un producto determinado (...)" (Premici, 2007) ${ }^{25}$.

Por otra parte, el 22 de Marzo de 2010 la Auditoría General de la Nación (AGN) presentó un informe de auditoría de gestión (Resolución $\mathrm{N}^{\mathrm{o}} 32 / 2010$ ) que evaluó las actuaciones de la FAN en el período comprendido entre el 31/08/05 y el 30/06/08. La AGN analizó tanto aspectos institucionales y organizativos como sustantivos y financieros.

Entre los aspectos institucionales y organizativos destacó que el Ministerio de Economía siguió participando en las decisiones de nombramiento pese a las prescripciones de la ley 26.338 , la falta de organigrama formal en la fundación, los incumplimientos a la hora de cubrir cargos fundamentales (Director General, Comité Ejecutivo), la falta de dinamismo para tomar decisiones, las infracciones en las contrataciones de personal, la ausencia de un mecanismo de control de horarios para el personal, y la falta de sistema de mesa de entradas y registración de tramitaciones internas.

En los aspectos sustantivos la auditoría destacó que la FAN no alcanzó ninguna de las metas anuales que se habían previsto, que sus convocatorias para ideas-proyecto no se registraban y tenían vicios formales y que los convenios suscriptos con entidades públicas y privadas carecían de graves irregularidades en su formación y en el control de su ejecución (contratos sin fecha, firmados por persona diferente del Director, etc.). 
Finalmente, en los aspectos financieros, la AGN expresó que en el trienio 2005/2007, ingresó a la FAN la suma de \$19.179.573. De esa suma el 93,91 $\%$ correspondió a aportes del Estado, 6,04 \% a ingresos financieros y el 0,05 $\%$ restante a ingresos propios. De esos ingresos se gastó el 3,90\% del total, es decir \$ 748.379, correspondiendo la mayor parte a gastos administrativos. La AGN concluyó que "los fondos aportados por el Estado a la FAN no fueron invertidos en los objetivos para los cuales fue creada" 26 .

\section{Las renegociaciones}

En Junio de 2005 la diputada Puig de Stubrin (UCR) -con otros legisladores- presentó un proyecto de ley que retomaba las críticas que formuló desde su lugar de presidenta de la comisión de Ciencia y Tecnología de la Cámara de Diputados (ver ut supra) ${ }^{27}$. Este proyecto proponía adecuar el diseño de las políticas de desarrollo de micro y nanotecnologías al esquema institucional vigente por ese entonces, previendo la intervención de la antigua Secretaría de Ciencia, Tecnología e Innovación Productiva de la Nación, que debía presentar el plan estratégico con un horizonte mínimo de diez años, con actualizaciones y rendiciones de cuentas trianuales al Congreso.

Asimismo se preveía que ese plan debía satisfacer las prescripciones de la ley 25.467, es decir, debía adecuarse al marco general de políticas científicas. También incluyó una serie de requisitos mínimos que el plan debía satisfacer, entre los que se contaban la priorización de las líneas que ofrecieran mejores "ventajas competitivas", la protección de las invenciones locales, la incubación de emprendimientos, etc. Respecto al financiamiento, se preveía reemplazar la discrecionalidad por mecanismos de concurso y evaluación de proyectos acorde a las pautas del sistema nacional de CyT, ley 25.467 y conexas.

Se reemplazaba la Fundación Argentina de Nanotecnología por el Fondo Argentino de Nanotecnología (misma sigla) dentro del Ministerio de Economía y Producción, y se regulaban las atribuciones de los órganos, el organigrama y la composición de su patrimonio ${ }^{28}$.

En los fundamentos de este proyecto se apreciaba una mirada más cautelosa hacia la política en el sector de las nanotecnologías ${ }^{29}$. Se expresaba que Argentina tiene una experiencia muy incipiente, y no dispone ni del equipamiento, ni del personal ni de las industrias con capacidad para el desarrollo 
de productos vinculados a la nanotecnología. A criterio de este grupo de diputados, los mecanismos que fija el Decreto 380/2005 parecen beneficiar sólo a un reducido grupo de investigadores que tienen contactos personales con una empresa extranjera particular.

Se decía que de continuar con el modelo de inversión del Decreto 380/2005 o de adoptar la institucionalidad propuesta por el proyecto 1357-D-05 (analizado ut supra), los recursos humanos formados no tendrían otra opción que emigrar al exterior, dado que el país "no contaría con una infraestructura para la producción de productos nanotecnológicos, ni habría puestos de trabajo en el sistema de C\&T o en el sector privado".

El contraproyecto no se hizo esperar. Los diputados Miguel Dante Dovena (FPV-PJ) y Jorge Raúl Giorgetti (FPV-PJ) presentaron en Diciembre de 2006 un proyecto de ley marco de la industria nanotecnológica de nueve $\operatorname{artículos}^{30}$. Una de las novedades respecto a los proyectos anteriores fue la incorporación al propio texto de algunas definiciones técnicas, en particular la de nanoproducto o nanocomponente, o la de industria nanotecnológica (ver infra). La autoridad de aplicación prevista era el Instituto Argentino de Nanotecnología (IANATEC), dentro de la órbita del organismo de mayor jerarquía en materia industrial del PEN, con autarquía operativa y financiera. Conforme a la tónica de los proyectos presentados por este bloque, el IANATEC se pensaba sometido a una fuerte influencia del Poder Ejecutivo (reglamentación, nombramientos, presupuesto, etc.).

En términos generales los fundamentos son similares a proyectos anteriores, aunque aparece por primera vez la consideración "que la totalidad de los avances está concentrada en los países más poderosos del mundo, lo que permite anticipar las dificultades que tendrá el conjunto de la población mundial para acceder a los beneficios de esta tecnología", pero con el temor que "aquellos países que no intervengan ni participen activamente en este proceso, verán ampliada la brecha con los más desarrollados, lo que a mediano plazo se traducirá en mayor exclusión social"31.

En el año 2008, el diputado Miguel Dante Dovena presentó un proyecto prácticamente similar al precitado ${ }^{32}$. Las únicas modificaciones respecto al texto de 2006 fueron el cambio de nomenclatura del proyectado Instituto Argentino de Nanotecnología (IANATEC) a Instituto Nacional de Nanotecno- 
logía (INNATEC), la composición de su Consejo Directivo y la forma de solventarlo. Los fundamentos de ambos proyectos tienen variaciones ínfimas, pero comparten tres cuestiones centrales: a) "que la adecuada política de comercialización de nuestros productos básicos o commodities (...), ha actuado y actúa de sostén social, evitando la profundización de la pobreza y permitiendo una sustancial mejora de la calidad de vida" (...); b) que "no podemos esperar que el crecimiento de nuestro país sea exclusivamente a base de exportar materia prima, que por definición es el comienzo en la cadena de valor de un producto manufacturado"; c) que se aprecia la "necesidad de ir a la búsqueda de productos con valor tecnológico agregado".

\section{Etapa 2007-actualidad. Los nano-objetos reordenando el espacio de lo público y lo privado}

El discurso de las nanotecnologías ya no podía sostenerse desde la estructura creada por el Decreto 380/2005 ni apelando al discurso de la biotecnología agrícola. En el año 2007 la SECyT desaparece y se crea el Ministerio de Ciencia y Técnica e Innovación Productiva (MINCyT). El MINCyT continuó considerando a las N\&N como prioritarias para el país junto con las TICS y la biotecnología y la FAN pasó a depender del nuevo ministerio ${ }^{33}$.

En 2008, se creó el Centro Interdisciplinario de Nanociencia y Nanotecnología (CINN), que reunía a cerca de 80 investigadores de las principales instituciones de Buenos Aires, La Plata y Bariloche. En el mismo año, se creó el Instituto de Nanociencia y Nanotecnología (INN) de la CNEA.

Cabe también señalar que en el Plan Nacional de Ciencia, Tecnología e Innovación "Argentina Innovadora 2020", la Nanotecnología es una de las tres Tecnologías de Propósito General consideradas como prioritarias, en particular por sus "múltiples aplicaciones posibles en diversas áreas como industria y agroindustria, ambiente, energía, salud y tecnologías sociales" (MINCyT, 2012a, 9) ${ }^{34}$.

La ANPCyT, a través del Fondo Nacional de Investigación Científica y Tecnológica (FONCyT), aprobó en el período 2000-2008 más de 160 proyectos relacionados con la nanotecnología (MINCyT, 2012b). En 2010, la ANPCyT inició el programa Fondos Argentinos Sectoriales (FONARSEC), al que solo podían aplicar consorcios público-privados. Este programa defi- 
nió dos grupos de fondos: los Fondos de Innovación Tecnológica Sectorial (FITS) y los Fondos Tecnológicos Sectoriales (FTS). Cabe también destacar en la ANPCyT los Aportes No Reembolsables Biotecnología, Nanotecnología y TIC (ANR BIO NANO TIC) del Fondo Tecnológico Argentino (FONTAR), que tienen por objeto mejorar las estructuras productivas y la capacidad innovadora de las empresas productoras de bienes y servicios del sector de las nanotecnologías (nanomateriales, nanointermediarios y nanosensores $)^{35}$.

La convocatoria de Fondos Sectoriales en Alta Tecnología de nanotecnología 2010 tuvo como objetivo financiar parcialmente proyectos orientados a generar plataformas tecnológicas o espacios para promover la innovación en el sector para así lograr el desarrollo de productos y/o tecnologías de aplicación general con potencial impacto en áreas productivas (MINCyT, 2013, 5) ${ }^{36}$.

En 2011 el MINCyT y la Comunidad Europea firman el acuerdo de cooperación internacional "Programa Fortalecimiento de la Competitividad de las PYMES y Creación de Empleo en la Argentina", que buscaba mejorar la competitividad del sector privado agregando conocimientos y valor a sus productos y servicios (cfr. Salvarezza, 2011).

En cuanto a las iniciativas legislativas de esta etapa, en Julio de 2014, los senadores nacionales Adolfo Rodríguez Saá (PJ San Luis) y Liliana Negre de Alonso (PJ San Luis) presentaron un proyecto que proponía un marco legal común para las nanotecnologías, la física cuántica y la biología sintética ${ }^{37}$. Se preveía la creación de un Instituto Nacional de Nanotecnología, Física Cuántica y Biología Sintética (INFIBI), como un ente autárquico dentro del ámbito del Ministerio de Ciencia, Tecnología e Innovación Productiva de la Nación. El proyecto reservaba para el INFIBI funciones de investigación, educación y extensión, y lo estructuraba en tres órganos (Comisión Asesora Nacional; Consejo Directivo Nacional; Dirección General Nacional), previendo asimismo la participación de todas las provincias y una cantidad apreciable de representantes académicos en la Comisión Asesora (el PEN sólo podía elegir un representante). Había en el articulado una clara disminución de la injerencia del PEN en el gobierno del ente, ya que el presidente de la Comisión podía ser cualquier integrante elegido por mayoría simple. Se preveía una eximición de derechos de aduana para todos los materiales (máquinas, libros, productos químicos, etc.) que debieran introducirse del extranjero para 
cumplir con los fines propuestos, y se proyectaban algunas modificaciones formales en la Ley 26.270 de promoción de la biotecnología moderna ${ }^{38}$.

\section{Las experiencias regulatorias}

En el campo de las N\&N, la idea clásica de regulación de una actividad o industria a través de la legislación ha sido reconceptualizada. Dalton-Brown $(2015,77)$, por ejemplo, reseña cuatro tipos de enfoques regulatorios. Primero, los registros (v.g. UK Voluntary Reporting Scheme, Swiss Nano-inventory). Segundo, los sistemas de gestión de riesgo, que pueden ser estatales (v.g. NanoKommission) o privados (v.g. NanoRisk Framework). Tercero, los códigos de conducta generados por las agencias del Estado, las ONG o los privados (v.g. IG-DHS Code of Conduct for Nanotechnology, BASF Code of Conduct Nanotechnology). Cuarto, la regulación vigente que pueda ser aplicada a las nanotecnologías (leyes ambientales, códigos civiles, etc.).

El formato de presentación de esta regulación, descentralizado e interdisciplinar, representa el reordenamiento del poder y de las prioridades en nuestras sociedades (Hodge et al, 2010, 10), por lo que la proliferación de nano-objetos es un desafío para las técnicas jurídicas tradicionales (Bailo, 2014, 105).

Como se anticipó ut supra, una de las primeras menciones locales a las necesidades regulatorias en materia de N\&N proviene de la Declaración sobre el Decreto 380/2005 que emitió el CECTE el 30 de Septiembre de 2005. En una nota a pie de página, el Comité declaró que en materia de nanotecnologías "junto a los posibles beneficios existen potenciales consecuencias cuyas implicaciones sociales deben preverse y analizarse".

Como explica Nelkin $(1975,35)$, a medida que ciertas tecnologías se perciben controversiales, los propios científicos, cuya experticia es la base de las decisiones técnicas, se ven envueltos también en discusiones públicas. De acuerdo al CECTE, los aspectos éticos y jurídicos de las N\&N fueron discutidos por grupos de expertos en dos eventos subsiguientes. En la Segunda Reunión Argentino-Brasileña "Ciencia, Tecnología y Sociedad", que tuvo lugar del 5 al 8 de Junio de 2006 en el Museo Argentino de Ciencias Naturales "Bernardino Rivadavia", se abordó el problema de las condiciones éticas del desarrollo de las N\&N. Lo propio tuvo lugar en las Primeras Jornadas 
"Ética en el Acceso al Conocimiento" que tuvieron lugar el 2 y 3 de octubre de 2006, en las que -entre otras cosas- se discutieron cuestiones vinculadas al patentamiento y las diferentes propiedades de las sustancias químicas conocidas en la macro y nanoescalas. Ambos eventos estaban vinculados al Programa Argentino-Brasileño de Ética en la Ciencia y la Tecnología rubricado en Noviembre de 2004 y que entró en vigencia en 2006.

En Agosto de 2006, la Secretaría de Ciencia, Tecnología e Innovación Productiva (SCTIP) dictó la Resolución 1084/2006, correspondiente al reglamento operativo de las diversas líneas de financiamiento del Programa de Modernización Tecnológica III ${ }^{39}$. El Anexo III del Reglamento, dedicado a la Gestión Socio-Ambiental, puso en cabeza de la Unidad de Gestión Socio-Ambiental - dependiente de la ANPCyT- el cumplimiento de los criterios ambientales ${ }^{40}$. El artículo 21 inciso 1 de este Anexo, expresa que el monitoreo y revisión socioambiental de proyectos incluirá aquellos más riesgosos, e incluye "tecnología de mecánica y materiales, incluyendo la nanotecnología, donde pueda haber impactos negativos ambientales o riesgos a la salud humana".

De esta manera, las instituciones que se apoyan en la autoridad científica de sus miembros vuelven colectivas las decisiones políticas relativas a la inserción de innovaciones científicas y tecnológicas en la sociedad (Bailo y Levrand, 2017, 63), aunque esta integración de expertos a la decisión pública y los formatos regulatorios alternativos a menudo no satisfacen los criterios democráticos (cfr. Desmoulin- Canselier y Lacour, 2011).

En Noviembre de 2007, el Ministerio de Salud dictó la Resolución 1490/2007, que aprobó la Guía de las Buenas Prácticas de Investigación Clínica en Seres Humanos. La norma contenía referencias a las nanotecnologías en el glosario especializado para medicina genómica-proteómica y medicina regenerativa, como una de las disciplinas que requieren validación científica en un marco ético apropiado. La norma define a la medicina regenerativa como una especialidad de naturaleza eminentemente interdisciplinaria, que incluye conceptos de ramas tan diversas "como la biología celular y molecular, la microfabricación, la robótica y ciencias de los materiales, particularmente a escala nano (10-9 unidades): nanotecnología, para diseñar partes de reemplazo del cuerpo humano". La norma fue reemplazada por la Resolución 1480/2011 del Ministerio de Salud, que aprobó una nueva Guía para Investigaciones con Seres Humanos, que no contiene referencias a las N\&N. 
En Agosto de 2008 una serie de expertos de distintas disciplinas se reunieron en el marco de la Conferencia Internacional para la Investigación Responsable en Nanociencia y Nanotecnología ${ }^{41}$ para discutir la posibilidad de adoptar un Código de Conducta en estas áreas ${ }^{42}$. De acuerdo a los registros del MINCyT, en esta Conferencia Lino Barañao enfatizó la necesidad de "prever las posibles consecuencias negativas de la Nanotecnología, así como también aprovechar el impacto de estas nuevas tecnologías para el desarrollo social y económico de la región". El ministro destacaba también que estos eventos "demuestran la preocupación del mundo científico por prevenir riesgos y por informar a la sociedad de manera adecuada a fin de que se puedan aprovechar plenamente los beneficios que ofrecen la nanociencia y la nanotecnología"-el resaltado nos pertenece- (MINCyT, 2008).

No obstante ello, los ESCT han puesto en duda que la resolución de los conflictos dependa de más investigación, mejor evidencia y mejor asesoramiento experto (Nelkin, 1987, 284), dado que "en las disputas políticas y jurídicas muchas veces es suficiente plantear preguntas críticas ante los expertos para así relativizar o incluso socavar su monopolio de experticia" (Stehr, 2013, 65).

En esta Conferencia se debatieron, entre otras cuestiones, el papel del principio precautorio y la evaluación fundada del balance entre los beneficios de las innovaciones y sus riesgos. Según el CECTE, la mayoría de las opiniones convergieron alrededor de: a) la necesidad de contribuir a crear una opinión pública informada; b) la importancia de evaluar los riesgos de los nanobjetos que llegan al mercado, tanto los de producción nacional como los que ingresan desde el exterior; c) impulsar políticas tendientes a la formación de especialistas en los conocimientos y técnicas específicas exigidas para analizar la toxicidad de nanobjetos. El Comité reseña también que en este evento se propuso la creación de un Observatorio de N\&N que accediera a bases de datos de investigaciones sobre riesgos en este campo y se discutieron las ventajas de contar con un código de ética en N\&N (cfr. BET, 2009, 3).

A raíz de ello, se acordó que el CECTE, con la participación de investigadores e industriales y de la FAN, iniciaría en el ámbito del MINCyT el proceso de elaboración de ese código. Para el CECTE, el código estaba pensado como una plataforma para el diálogo sobre la $\mathrm{N} \& \mathrm{~N}$ entre instituciones científicas $y$ de financiamiento e industriales con el resto de la sociedad destinado a in- 
formar y favorecer la confianza social en la investigación. En el mismo sentido, el CABNN en distintas reuniones ha propuesto avanzar en la difusión de un Código de Ética nano, crear un "Observatorio Nano" e incluir la temática de riesgos en la enseñanza de las $\mathrm{N} \& \mathrm{~N}$ y en el diseño de los proyectos.

La falta de consenso respecto de las definiciones fundamentales en el campo de las N\&N ha hecho que las organizaciones que se dedican a la estandarización se interesaran especialmente en clarificar el lenguaje a emplear (cfr. Hodge et al, 2010, 2). En 2005, con la finalidad de avanzar en la estandarización en el campo de las nanotecnologías, la Organización Internacional de Normalización (ISO) creó el Comité Técnico ISO/TC 229. El Comité cuenta con 37 países en calidad de participantes y 14 en calidad de observadores, y lleva publicadas 53 normas estandarizadas ${ }^{43}$. Cuenta también con varios subgrupos de trabajo según el área de incumbencia y la colaboración que recibe de otras organizaciones ${ }^{44}$. Las organizaciones de estandarización entienden a las normas como documentos técnicos que representan el estado de la ciencia y de las mejores prácticas en un momento dado, y contienen información consensuada por todas las partes interesadas. Para algunos autores el criterio escalar que adoptó ISO se traduce en una estandarización que se presenta como "hecha por la ciencia" para asegurar que los objetos circulen en mercados globales (Laurent, 2017, 96).

Argentina participa en el Comité ISO/TC 229 como país observador a través del Instituto Argentino de Normalización y Certificación (IRAM). En 2008, el IRAM creó un comité de nanotecnologías que está conformado por especialistas locales de diversas disciplinas, y sigue, como miembro observador, los avances en materia de normalización técnica del ISO/TC 229. El comité tiene como función principal establecer políticas y líneas de acción para el desarrollo de la normalización en el área de las nanotecnologías. El Subcomité de nanotecnologías que funciona en el mismo Comité, tiene como función el desarrollo de normas en el campo de las tecnologías que se desarrollan en la escala nanométrica, en particular, terminología; medición y caracterización; salud, seguridad y aspectos ambientales; y especificación de materiales.

En materia de nanotecnologías, el IRAM dispone de dos normas catalogadas como $\mathrm{CGN}^{45}$, la norma 39501 sobre vocabulario y la 39502, de hoja de datos de seguridad; y dos normas catalogadas como $\mathrm{ANT}^{46}$, la norma 39503, de metodología para la evaluación del riesgo de nanomateriales y la norma 
39504, de gestión del riesgo ocupacional aplicado a nanomateriales de ingeniería, principios y enfoque basado en control de bandas ${ }^{47}$. El alcance de la estandarización que se realiza desde los organismos de normalización abarca tanto el entendimiento y control de los procesos que ocurren a nanoescala como la utilización de las propiedades de esos materiales ${ }^{48}$.

Las definiciones que traen los organismos de estandarización pueden obliterar del plano técnico los intereses que provienen de las audiencias locales. En ese sentido, los dispositivos de participación pública no son ajenos a las controversias acerca de las nanotecnologías. En Argentina la discusión por los productos de las N\&N no ha producido modificaciones en el derecho de fondo (v.g. códigos de derecho privado, normas ambientales y de consumo), como sí ha sucedido, por ejemplo, en Francia. Este país, luego de haber organizado un debate público sobre nanotecnologías en 2010, incorporó a través de distintas reformas a su Código del Medioambiente (Code de l'environnement) mecanismos de prevención de riesgos resultantes de la exposición a "sustancias en estado nanoparticulado" (substances à l'état nanoparticulaire) en los artículos 523-1/8. El Código consagró dos tipos de obligaciones para las personas que fabrican, importan o distribuyen estas sustancias. La primera es la obligación de declarar a la autoridad administrativa la identidad, calidad y usos de estas sustancias y la identidad de los usuarios involucrados en su manipulación, con el fin de garantizar la trazabilidad y la información al público. La segunda es que, a pedido de la autoridad administrativa, proporcionen información respecto de los peligros, potenciales exposiciones, y toda información útil para la evaluación de riesgos a la salud o al ambiente ${ }^{49}$.

Lo que hace complejas estas regulaciones es la propia inestabilidad de los nano-objetos. El Boletín Estadístico Tecnológico (BET) no 3 de 2009 del MINCyT expresó que "una de las principales dificultades para analizar los posibles riesgos de la nanotecnología es que se trata de un término global y que no se emplea a una sola tecnología o aplicación" (3). Este estudio reconoce que pueden existir riesgos con el medio ambiente y la salud asociados con la emisión no regulada de algunas nanopartículas de diseño durante el desarrollo, la fabricación, incorporación, uso o eliminación de productos, pero también pone de manifiesto el peligro implícito que tendrían algunas nanopartículas "creadas por la naturaleza". 
Según el Boletín, aunque la mayoría de estas inquietudes aún no tienen respuesta y continúan bajo debate, "se ha planteado la necesidad de generar un marco regulatorio seguro y responsable así como también la importancia de informar al público sobre los posibles beneficios y peligros de la nanotecnología" (2009, 3). El documento reitera el compromiso asumido en 2008 de avanzar en un Código de Ética para la Investigación en Nanotecnología, siguiendo las recomendaciones de la Unión Europea.

En un estudio ejecutado en 2012, llevado adelante por el MINCyT, sobre empresas y grupos de I+D de nanotecnología en Argentina, también se abordaron algunos aspectos legislativos. Según el estudio "los grupos de I+D realizaron pocas menciones referidas a este tema" (2012b, 31), aunque se registraron algunas preocupaciones respecto a la disposición final de nanopartículas y de los productos terminados que las contienen $(2012 \mathrm{~b}, 31)^{50}$.

De acuerdo al documento, cuando las empresas hacen referencia al tema lo ven como otro de los obstáculos para el progreso de la actividad ${ }^{51}$. En cuanto a las omisiones concretas, el estudio destaca el problema de la cobertura laboral del manejo de nanomateriales (que podrían tener efectos tóxicos) y la existencia de vacíos en los códigos aduaneros que no tienen aún clasificados distintos productos nanotecnológicos.

A raíz de una convocatoria del MINCyT, entre Octubre de 2012 y Diciembre de 2013, se realizó un estudio de prospectiva y vigilancia tecnológica en el sector nanotecnológico ${ }^{52}$. El estudio - publicado en 2016- entendió a la legislación, normativa o regulación como "cuello de botella", definido éste como factor limitante para el desarrollo del área de las N\&N $(2016,70)$, especialmente para mercados futuros de productos basados en nanotecnología que podrían exportarse $(2016,72)^{53}$.

El estudio resalta las dificultades que irroga la identificación de nanoproductos por lo que sugiere resolver con urgencia la discusión sobre adaptar las regulaciones o crear regulaciones totalmente nuevas, y la necesidad de etiquetado específico de los productos nanotecnológicos ${ }^{54}$. En este sentido, estableció que "se requieren métodos específicos que permitan evaluar los riesgos para la salud laboral de quienes trabajan con nanomateriales, por un lado y los que conlleva el uso generalizado de nanoproductos para la salud pública y medioambiental, por otro" (MINCyT, 2016, 193) $)^{55}$. 
El estudio también realizó consideraciones sobre la percepción social de los riesgos de las N\&N. Para los responsables de la consulta, la oportunidad de contrarrestar estas amenazas "estará en todo caso relacionada con la capacidad de aprovechar las posibilidades de proveer de mejoras significativas en el acceso a los recursos básicos como el agua potable, la energía, la salud, etc., a la población" (MINCyT, 2016, 194).

El proyectó arrojó como resultado una plataforma web para la gestión de la vigilancia tecnológica y del entorno: Vigiale Reporter PLUS, que permite el seguimiento de fuentes de información seleccionadas y la notificación de cambios detectados ${ }^{56}$. En la lógica del estudio, estas plataformas son útiles para espacios dónde las organizaciones, la sociedad civil y todos los grupos potencialmente afectados "puedan discutir, así como mantener, paralelamente, una actividad de benchmarking y vigilancia de los progresos y evolución de las regulaciones y normativa en el mundo, que permitan la anticipación" (MINCyT, 2016, 199).

De allí que una vez instalados en el sistema legal, los objetos de la tecnociencia pueden pasar por varias categorías jurídicas y moverse con facilidad por distintos perímetros legales (cfr. Bailo, 2014). Esto hace que muchos reguladores se pregunten si las $\mathrm{N} \& \mathrm{~N}$ pueden aprovechar los marcos normativos vigentes -por ejemplo en materia de identificación y control de productos- o si éstos demandan procesos ad hoc de revisión y adaptación.

En este sentido, en el año 2012 la Administración Nacional de Medicamentos, Alimentos y Tecnología Médica (ANMAT), en el marco del Observatorio ANMAT conformó un grupo de trabajo multidisciplinario, con el objeto de abordar y analizar aspectos vinculados a la aplicación de la nanotecnología en los productos para la salud de competencia del organismo ${ }^{57}$. Por otra parte, en Abril de 2013, la Superintendencia de Riesgos de Trabajo (SRT) dictó la Resolución 770/2013, que creó el Programa Nacional de Prevención por Rama de Actividad. El artículo 1 consagró como objetivo principal del Programa "el desarrollo y programación de políticas activas de prevención primaria, secundaria y terciaria, de manera conjunta o independiente con las Organizaciones Empresariales, las Organizaciones Sindicales, las Administradoras de Trabajo Locales y las Aseguradoras de Riesgo de Trabajo”. El artículo 3 de la Resolución incluyó expresamente a las nanotecnologías como una de las ramas de actividad incluidas en el Programa. 
En Septiembre del mismo año, el MINCyT lanzó Antena Tecnológica, una plataforma de vigilancia tecnológica e inteligencia competitiva (VTeIC) que tiene como objetivo brindar información para planificar y formular estrategias tecnológicas minimizando la incertidumbre del contexto dentro de las empresas e instituciones. Fue desarrollada de manera conjunta a través del Programa Nacional de Vigilancia Tecnológica e Inteligencia Competitiva (VINTEC) por la Secretaría de Planeamiento y Políticas del Ministerio y la Unión Industrial Argentina (UIA), y cubre diversas áreas de las N\&N como ser agroalimentación, energía, medicina, TICs, electrónica y nanomateriales (MINCyT, 2013). La Antena permite obtener boletines electrónicos y recopilar noticias que incluyen, entre otras cosas, información relativa a legislación y normativas legales y técnicas.

Finalmente, en Junio de 2017, el Ministerio de Ambiente y Desarrollo Sustentable (MAyDS) organizó en el marco de la Semana del Ambiente una serie de charlas de nanotecnología y ambiente en la que estuvieron involucrados expertos de distintas disciplinas. El evento tuvo por objetivo "concientizar y sensibilizar sobre la importancia de la Nanotecnología en cuanto a sus innovaciones y sus riesgos, pensándola como una herramienta que debe ser utilizada sustentablemente" (MAyDS, 2017).

\section{La polisemia de la nano-objetualidad}

Como se expresó anteriormente, estos vaivenes regulatorios están vinculados a la propia inestabilidad de los nano-objetos y a su plasticidad para reordenar las redes y reorientar los recursos comprometidos en su sostenimiento. El criterio para deslindar nano/no nano en los planos regulatorios no siempre responde al significado escalar del prefijo, ya que aquello que es válido para la ciencia puede no serlo para la política científica (cfr. Desmoulin-Canselier y Lacour, 2011 $)^{58}$. Como expresa Laurent $(2017,94)$, los programas científicos recurren al criterio del tamaño para demarcar lo que es menor y mayor que lo nano, lo que ha resultado insatisfactorio, por ejemplo, para identificar sustancias riesgosas, en las que el riesgo debe ser testeado para cada una en particular.

Definir a las nanotecnologías no es una mera tarea clasificatoria, sino una coproducción técnico-política en la que las capacidades y modos de decisión se distribuyen en las audiencias de expertos, funcionarios políticos, empresarios y ciudadanos en general. 
El Decreto 380/2005 no incluyó definiciones, pero aludía indistintamente a las micro y nanotecnologías y a su aplicación y desarrollo, lo cual encuentra explicación - más que en un criterio técnico- en la intención de incluir en la norma las investigaciones que venían realizando distintos organismos nacionales (INTI, INIFTA, CNEA, etc. ${ }^{59}$. Pueden citarse dos casos representativos de expertos que intervinieron activamente en las redes de $\mathrm{N} \& \mathrm{~N}$ y en las principales agencias científicas del país para estabilizar esas tensiones. Por un lado, el Dr. en Bioquímica Roberto Salvarezza, que fue responsable de la creación del Laboratorio de Nanoscopías en el Instituto de Investigaciones Fisicoquímicas Teóricas y Aplicadas (INIFTA) en 1992, que coordinó el Centro Argentino-Brasileño de Nanociencia y Nanotecnología, integró el Consejo de Administración de la Fundación Argentina de Nanotecnología y presidió el Consejo Nacional de Investigaciones Científicas y Técnicas (CONICET) en el período 2012-2015. Luego de su renuncia a este organismo, regresó a su lugar como Director de los laboratorios de Nanoscopías y Fisicoquímica de Superficies en el INIFTA. Por otro lado, el Ing. Electromecánico Daniel Lupi, que fue Director del Centro de Investigación en Telecomunicaciones Electrónica e Informática del INTI en el período 19952005, y se desempeña como Presidente de la FAN desde 2011.

En una entrevista correspondiente al programa $\mathrm{N}^{\mathrm{o}} 464$ de Ciencia Argentina en la Vidriera (2013) Salvarezza expresaba que a principios de los 90, estudiando en España con un microscopio de efecto túnel, "ver átomos y poder seguir las reacciones en tiempo real, me atrajo sobremanera". Surge de la entrevista que, si bien la nanoescala no era una novedad para Salvarezza, sí lo era "comprender la conveniencia de trabajar en conjunto con otras disciplinas para resolver temas complejos". Por otra parte, en una entrevista realizada por N. Luna (2015) -Agencia TSS- a Daniel Lupi, el ingeniero expresaba que "los chips con los que trabajábamos los ingenieros se fueron achicando tanto que, de repente, nos encontramos hablando de nanómetros y tuvimos que averiguar qué eran".

Ambos casos son demostrativos de un momento clave en la política científica sobre N\&N en Argentina, en tanto Salvarezza representa los esfuerzos de articulación y mutación de la investigación básica a la aplicada y Lupi, que provenía de la microelectrónica, representa los esfuerzos de adaptación de las disciplinas tradicionales. Estas tensiones entre investigación básica/aplicada y objetualidad micro/nano estuvieron presentes desde el 
mismo momento de constitución de la FAN y se mantuvieron en instrumentos políticos subsiguientes.

El proyecto 1357-D-05 definía en sus fundamentos a las nanotecnologías como "el conjunto de técnicas desarrolladas para la manipulación de objetos cuyas dimensiones son del orden del nanómetro (una millonésima de milímetro)". El proyecto 3279-D-05, en cambio, intentó incluir en sus fundamentos una definición que aunara micro y nanotecnologías, como "tecnologías destinadas al estudio, diseño, creación, síntesis, manipulación y aplicación de materiales funcionales y sistemas de control de la materia". Sin embargo, en cuanto al criterio escalar el concepto no era preciso pues las incluía "a escalas del nanómetro (la mil millonésima parte de un metro) o tecnologías que tienen tamaños comparables a la longitud de una pequeña molécula".

El proyecto 7277-D-2006 fue el primero que pretendió introducir definiciones técnicas en el articulado. Este proyecto, que no mencionaba a las microtecnologías, entendía a la nanotecnología a partir de un criterio escalar, como "el estudio, diseño, creación, síntesis y aplicación de materiales, aparatos y sistemas funcionales a través de la manipulación de la materia en dimensiones del orden del nanómetro, es decir, a nivel de átomos y moléculas". Sin embargo, el proyecto también traía conceptos que ampliaban considerablemente ese limitado criterio de operatividad. Se mencionaba a los nanoproductos o nanocomponentes, definiéndolos como "aquellos elaborados u obtenidos mediante el uso de herramientas y procedimientos desarrollados por la nanotecnología" y a la industria nanotecnológica como aquella que comprende "todos los procesos productivos involucrados en la fabricación de nanocomponentes, ya sea como producto final o como parte de un producto o proceso de mayor complejidad".

El análisis de las posibilidades de Argentina en el concierto global fue especificando el tipo de nano-objetos en el que los investigadores e inversores debían concentrar sus esfuerzos. Daniel Lupi, que ya había realizado algunas apreciaciones sobre la política pública temprana en nanotecnologías (ver ut supra), accede a la presidencia de la FAN en 2011. Para el ingeniero electromecánico, "la nanotecnología es una tecnología disruptiva y revolucionaria pero que en casi todos los casos lleva a modificaciones evolutivas en los productos existentes y no a nuevos 'nanoproductos'" (Lupi, 2012, 45; cfr. 
Mi Club Tecnológico, 2015). El presidente de la FAN distinguía, al principio de la cadena de valor los nanomateriales -donde dominan las grandes multinacionales químicas-, luego los nanointermediarios - donde creía que podría ubicarse la industria local-y luego los productos cotidianos mejorados por el empleo de los nanointermediarios (Lupi, 2012, 45; cfr. Luna, 2015) ${ }^{60}$.

A medida que las redes y plataformas locales de N\&N se consolidaban y los planes nacionales redefinían la impronta de las convocatorias y el financiamiento, los nano-objetos fueron segmentando su grafía para cubrir mayores terrenos y definir nuevas fronteras de colaboración entre lo público y lo privado. El BET (MINCyT, 2009, 2), partiendo de un análisis sobre la cadena de valor de las nanotecnologías, identificó cuatro eslabones. Los nanomateriales, como estructuras de la materia desarrolladas artificialmente con dimensiones inferiores a los 100 nanómetros que exhiben propiedades dependientes del tamaño y que han sido mínimamente procesadas. Los nanointermediarios, como productos intermedios que no caen en la categoría de nanomateriales ni de productos de consumo final, que incorporan nanomateriales o que han sido construidos con características nanométricas. Los productos nanoenriquecidos, como productos del final de la cadena de valor que incorporan nanomateriales o nanointermediarios. Por último, las nanoherramientas, como instrumentos técnicos y software utilizados para visualizar, manipular y modelar la materia a escala nanométrica.

Las tensiones entre investigación básica/aplicada y entre objetos micro/nano se estabilizaron cuando las redes estuvieron en condiciones de enfocarse más en la superficie que en la escala de los nano-objetos. Como lo expresa Lupi: "algo no es nanométrico sólo porque mide 1 o 2 nanómetros, sino porque en esa instancia las propiedades físicas y químicas de los materiales comienzan a tener una ambivalencia interesante y aparecen propiedades que antes no se evidenciaban" (Revista La Universidad, 2011, 31).

En ese sentido, los proyectos legislativos posteriores optaron por incluir definiciones más flexibles, que pretendían acompañar las tendencias de las redes especializadas. Por ejemplo, en los fundamentos del proyecto 2617-D2010 se decía que la nanotecnología "es la manipulación de la materia en la escala de los átomos y las moléculas (...)". Es una forma de producción "que concibe los bienes desde lo más chico hacia lo más grande, donde se utilizan los componentes necesarios para obtener lo que se necesita". De la misma 
manera, el proyecto 2092-S-14, pretendía definirla en su artículo 2 inc. 1 como el estudio de la capacidad técnica "para modificar y manipular la materia en las dimensiones más pequeñas conocidas, con el objeto de obtener materiales y productos a partir del reordenamiento de átomos y moléculas; y de desarrollar estructuras o dispositivos funcionales a las dimensiones nano".

En este camino crítico de la especificación de la nano-objetualidad, la polisemia no se reduce a un problema de múltiples acepciones. Los nano-objetos proponen sus propias metas políticas y regulan sus propios modos de alcanzarlas. Por ejemplo, los responsables de los sucesivos lanzamientos de nano-satélites argentinos al espacio han manifestado en reiteradas oportunidades que estos desarrollos buscan la democratización del acceso al espacio (MINCyT, 2013b) y de la tecnología espacial (MINCyT, 2013c).

\section{Algunas conclusiones provisorias}

Como se advirtió a lo largo del artículo, una propuesta de co-creación de la nano-objetualidad exige revisitar los distintos tipos de conocimientos comprometidos en la tarea de regulación y de construcción de la institucionalidad pública. Los nano-objetos se agencian del mundo jurídico y aprenden su lenguaje, por lo que una vez instalados en el sistema pueden moverse con facilidad por distintos perímetros legales.

Esos vaivenes regulatorios están vinculados a la propia inestabilidad de los nano-objetos y a su plasticidad para reordenar las redes y reorientar los recursos comprometidos en su sostenimiento. Cuando las redes de lo jurídico asumen que los objetos de la tecnociencia están demarcados, la actividad regulatoria se entiende como un proceso de construcción vertical de reglas. Cuando asumen que esos objetos son inestables y controvertidos, la regulación se entiende como un ejercicio coordinado de estabilización. En ese ejercicio de coproducción técnico-política de la nano-objetualidad los derechos de participación en las decisiones públicas y las garantías de acceso al conocimiento se convierten en dos indicadores clave de las prácticas democráticas.

En Argentina, los nano-objetos han tenido dos puertas de entrada al mundo regulatorio. Por un lado, el proceso que llevó a la creación de la FAN en 2005 que entendía la regulación como la reglamentación de una tecnología que podía transformar el perfil productivo del país. Los distintos proyec- 
tos legislativos de reforma que se han presentado en el período 2005-2014 no han cuestionado que el derecho deba facilitar la inserción de Argentina en ese panorama global, sino que han propuesto otros medios para lograrlo. Por otro lado, el proceso que inicia con la ex SECyT y continúa con el actual MINCyT, que ha entendido la regulación como un ejercicio de estabilización de las distintas tensiones que acompañan a los nano-objetos (local/global; investigación básica/aplicada; micro/nano; escala/superficie). Ello motivó que finalmente el MINCyT absorbiera la institucionalidad de la FAN y se convirtiera en el epicentro de las redes que coproducen el gobierno nano en Argentina. Para consolidar este dispositivo el MINCyT se apoyó en las distintas iniciativas de la ANPCyT, en la reordenación de los espacios público y privado de las redes de investigación y desarrollo de las N\&N, en concentrar la organización de distintas reuniones de expertos y propuestas de mecanismos regulatorios a través del CECTE, en la actividad de difusión y formación que hace la FAN, en fortalecer los procesos de vigilancia y obtención de información clave en el sector, $\mathrm{y}$ fundamentalmente en administrar la polisemia de la nano-objetualidad hacia el interior de las redes y a través de los distintos actores clave del sector.

No obstante ello, el gobierno de lo nano en Argentina encierra otras tensiones y desestabilizaciones. Las distintas iniciativas para lograr un observatorio nano interdisciplinario y participativo, para avanzar en la diagramación de códigos de ética y para iniciar una revisión de la eficacia de los marcos normativos vigentes todavía no ha tenido resultados apreciables. De la misma manera, para favorecer la circulación de los nano-objetos, Argentina ha avanzado en la sincronización de sus espacios y redes con la estandarización técnica global, lo cual complejiza el diálogo entre las experiencias aprendidas y los espacios globales. Finalmente, la ciudadanía no se ha involucrado significativamente en el gobierno y en las discusiones derivadas de las N\&N.

Todo lo expuesto invita a pensar que la grafía de los nano-objetos aguarda nuevas inscripciones que orienten las prácticas científicas y sociales a una mayor democratización de las N\&N en Argentina. 


\section{Referencias}

Andrini, L. y Figueroa, S. (2008). El impulso gubernamental a las nanociencias y nanotecnologías en Argentina. En G. Foladori y N. Invernizzi (Eds.), Las nanotecnologías en América Latina. México DF: Porrúa.

Bailo, G. (2014). Estrategias jurídicas locales para el gobierno de los nanoresiduos. Construyendo una agenda jurídica regulatoria para nanomateriales en el derecho argentino. Revista Jurídica de Derecho Ambiental, (38), 103-126.

Bailo, G. L., y Levrand, N. E. (2017). Casos controversiales: un estudio de la relación entre ciencia y derecho en el proceso judicial. Estudios SocioJurídicos, 20(1), 45-74. http://dx.doi.org/10.12804/revistas.urosario. edu.co/sociojuridicos/a.5341

Dalton-Brown, S. (2015). Nanoregulation. En Nanotechnology and Ethical Governance in the European Union and China (pp. 73-101). Cham: Springer.

Desmoulin-Canselier, S. y Lacour, S (2011). Chapter 29: Nanotechnology and the Law. En: P. Houdy, M. Lahmani y F. Marano (Eds.) Nanoethics and Nanotoxicology. Berlin: Springer.

Fiorani, V. (2011). Normas para una industria próspera y segura: Nanotecnologías. Boletín del Instituto Argentino de Normalización y Certificación (IRAM), 2(2), 12-13.

Foladori, G. (2016). Políticas públicas en nanotecnología en América Latina. Problemas del desarrollo, 47(186), 59-81. https://doi.org/10.1016/j.rpd. 2016.03.002

Hermitte, M. A. (2007). Fundación jurídica de una sociedad de las ciencias y de la técnica a través de las crisis y los riesgos. En Derecho, sociedad y riesgos: la sociedad contemporánea vista a través de la idea de riesgo. Red Latinoamericana - Europea sobre Gobierno de los Riesgos, UniCEUB, UNITAR. Brasilia. 
Hodge, G. A., Bowman, D. M. y Maynard, A. D. (2010). International handbook on regulating nanotechnologies. Cheltenham, UK: Edward Elgar.

Hubert, M. (2014). Modelo dominante y variaciones nacionales en el diseño de políticas de investigación en favor de la innovación tecnológica: una aproximación comparativa de los dispositivos de apoyo a la nanociencia y nanotecnología en Argentina y Francia. Estudos de Sociología, 19(37), 391-408.

Hubert, M. y Spivak L'Hoste, A. (2009). Integrarse en redes de cooperación en nanociencias y nanotecnologías: el rol de los dispositivos instrumentales. Redes, 15(29), 69-91.

Hurtado, D., Lugones, M. y Surtayeva, S. (2017). Tecnologías de propósito general y políticas tecnológicas en la semiperiferia: el caso de la nanotecnología en la Argentina. Revista Iberoamericana de Ciencia Tecnología y Sociedad, 12(34), 65-93.

Invernizzi, N., Hubert, M. y Vinck, D. (2014). 11: Nanoscience and Nanotechnology: How an Emerging Area on the Scientific Agenda of the Core Countries Has Been Adopted and Transformed in Latin America. En: E. Medina et al. (ed.) Beyond Imported Magic. Essays on Science, Technology, and Society in Latin America (pp. 223-242). Cambridge, Massachusetts: MIT Press.

Jasanoff, S. (2011). Momentos constitucionales en el gobierno de la ciencia y la tecnología. En T. Pérez Bustos y M. Lozano Borda (Eds.), Ciencia, tecnología y democracia: reflexiones en torno a la apropiación social del conocimiento. Medellín: Colciencias-Universidad Eafit.

Laurent, B. (2017). Democratic Experiments: Problematizing Nanotechnology and Democracy in Europe and the United States. Cambridge, Massachusetts: MIT Press.

Lupi, D. (2012). Nanotecnología e industria argentina. Voces en el Fénix, $3(16), 42-49$. 
Nelkin, D. (1975). The political impact of technical expertise. Social Studies of Science, 5(1), 35-54.

Nelkin, D. (1987). Controversies and the authority of science. En H. Engelhardt y A. L. Caplan (Eds.), Scientific controversies: case studies in the resolution and closure of disputes in science and technology (pp. 283293). Nueva York: Cambridge University Press.

Nelkin, D. (1995). Science controversies the dynamics of public disputes in the United States. En S. Jasanoff (Ed.), Handbook of science and technology studies. Londres: SAGE.

Revista La Universidad (2011). Entrevista al Ing. Daniel Lupi (Presidente de la Fundación Argentina de Nanotecnología). Revista La Universidad, Universidad Nacional de San Juan, 8(52), 30-31.

Salvarezza, R. (2011). Situación de la difusión de la nanociencia y la nanotecnología en Argentina. Mundo Nano, (4)2, 18-21.

Stehr, N. (2013). Las sociedades modernas del conocimiento. En: AA. VV. La sociedad de la información y del conocimiento. $\mathrm{N}^{\circ} 11$ (pp. 57-67). Santiago de Chile: Konrad Adenauer-Stiftung.

Vila Seoane, M. F. (2014). Los desafíos de la nanotecnología para el "desarrollo" en Argentina. Mundo Nano, 7(13), 78-94.

\section{Normativa citada}

Argentina. Decreto Poder Ejecutivo Nacional No 380/2005. Aplicación y desarrollo de micro y nanotecnologías. Publicada en el Boletín Oficial del 29-abr-2005, Número 30643, Página 5.

Argentina. Ley Nacional No 24.156. Administración Financiera y Sistemas De Control. Disposiciones Generales. Publicada en el Boletín Oficial del 29-oct-1992, Número 27503, Página 1. 
Argentina. Ley Nacional No 25.467. Ciencia, Tecnología e Innovación. Sistema Nacional - Régimen Legal. Publicada en el Boletín Oficial del 26sep-2001, Número 29740, Página 1.

Argentina. Ley Nacional No 26.338. Ley de Ministerios. Publicada en el Boletín Oficial del 07-dic-2007, Número 31298, Página 3.

Argentina. Ministerio de Economía y Producción. Resolución 579/2005. Fundación Argentina de Nanotecnología - ampliase cantidad de miembros. Publicada en el Boletín Oficial del 27-oct-2005, Número 30768, Página 15.

Argentina. Ministerio de Relaciones Exteriores, Comercio Internacional y Culto (M.R.E.C.I. Y C.). Protocolo para la Creación del Centro Argentino Brasileño de Nanotecnología (CABN) entre la República Argentina y la República Federativa del Brasil. Publicado en el Boletín Oficial 10/01/2006.

Argentina. Ministerio de Salud. Resolución 1490/2007. Salud Pública. Guía de las Buenas Prácticas de Investigación Clínica - Aprobación. Publicada en el Boletín Oficial del 14-nov-2007, Número 31281, Página: 6. Abrogada por el Artículo $6^{\circ}$ de la Resolución 1480 Del Ministerio De Salud, B.O. 21/09/2011.

Argentina. Ministerio de Salud. Resolución 1480/2011. Salud Pública. Guía para Investigaciones con Seres Humanos - Aprobación. Publicada en el Boletín Oficial del 21-sep-2011, Número 32239, Página 14.

Argentina. Secretaría de Ciencia, Tecnología e Innovación Productiva. Resolución 1084/2006. Programa de Modernización Tecnológica III. Reglamento Operativo - Su Aprobación. Publicada en el Boletín Oficial del 15-ago-2006, Número 30969, Página 22.

Argentina. Superintendencia de Riesgos del Trabajo. Resolución 770/2013. Programa Nacional de Prevención por Rama de Actividad-Créase. Publicada en el Boletín Oficial del 06-may-2013, Número 32632, Página 9. 


\section{Proyectos legislativos citados}

Argentina. Proyecto de Ley Número de Expediente 264-S-01. Proyecto de ley sobre elaboración del plan estratégico de ciencia y tecnología. Autor: Moreau, Leopoldo. Origen: Senado de la Nación. Publicado en: DAE 017/01. Fecha: 03/04/2001.

Argentina. Proyecto de Ley Número de Expediente 1357-D-05. Creación del Instituto Argentino de Nanotecnología, en el ámbito del Ministerio de Economía. Autor: Giorgetti, Jorge. Publicado en: Trámite Parlamentario no 21. Fecha: 31/03/2005.

Argentina. Proyecto de Resolución Número de Expediente 2844-D-2005. Pedido de informes al Poder Ejecutivo sobre los objetivos de las políticas cientificas y la creación de la Fundación Argentina de la Nanotecnología (Resolución del ME NS 380/05). Firmantes: Puig De Stubrin, Lilia; Beccani, Alberto; Borsani, Luis; Chironi, Fernando; Giubergia, Miguel; Iglesias, Roberto. Publicado en: Trámite Parlamentario $\mathrm{N}^{\circ}$ 52. Fecha: $13 / 05 / 2005$.

Argentina. Proyecto de Ley Número de Expediente 3279-D-2005. Plan Nacional Estratégico de Desarrollo de Micro y Nanotecnologías, y derogación del Decreto 380/05. Autores: Puig De Stubrin, Lilia; Chironi, Fernando; Rodríguez, Marcela; Larreguy, Carlos; Beccani, Alberto; Cettour, Hugo; Giubergia, Miguel; Borsani, Luis. Publicado en: Trámite Parlamentario $n^{\circ}$ 65. Fecha: 02/06/2005.

Argentina. Proyecto de Resolución Número de Expediente 5416-D-2005. Pedido de Informes al Poder Ejecutivo sobre el Financiamiento de Proyectos de Investigación Científica, Desarrollo Tecnológico o Innovación Productiva. Autores: Puig de Stubrin, Lilia; Storani, Federico; Negri, Mario. Publicado en: Trámite Parlamentario nº 132. Fecha: 16/09/2005. 
Argentina. Proyecto de Resolución Número de Expediente 0753-D-2006. Pedido de Informes al Poder Ejecutivo sobre el Financiamiento de la Conferencia de Carácter Cientifico Realizada en el Hotel Tunquelen, Bariloche, Provincia de Rio Negro, por parte de la Oficina de Investigaciones Navales y de la Fuerza Aérea de Estados Unidos de América. Autores: Rodríguez, Marcela; García, Susana; Bisutti, Delia. Publicado en: Trámite Parlamentario no 13. Fecha: 17/03/2006.

Argentina. Proyecto de Ley Número de Expediente 7277-D-2006. Ley Marco de la Industria Nanotecnológica. Autores: Dovena, Miguel Dante y Giorgetti, Jorge Raúl. Publicado en: Trámite Parlamentario n ${ }^{\circ} 188$. Fecha: 07/12/2006.

Argentina. Proyecto de Ley Número de Expediente 4853-D-2008. Ley marco de la industria nanotecnológica (INNATEC): objetivo, definiciones, autoridades. Autor: Dovena, Miguel Dante. Publicado en: Trámite Parlamentario $N^{\circ} 114$. Fecha: 04/09/2008

Argentina. Proyecto de Resolución Número de Expediente 2617-D-2010. Pedido de informes al poder ejecutivo sobre diversas cuestiones relacionadas con el funcionamiento de la "Fundación Argentina de Nanotecnología", dependiente del Ministerio de Ciencia y Tecnología e Innovación Productiva. Firmantes: Rioboo, Sandra; Alvarez, Elsa; Fiad, Mario; Veaute, Mariana; Storani, Maria Luisa; Juri, Mariana; Mansur, Ricardo. Publicado en: Trámite Parlamentario No 43. Fecha: 28/04/2010.

Argentina. Proyecto de Resolución Número de Expediente 3353-D-2010. Pedido de informes al Poder Ejecutivo sobre diversas cuestiones relacionadas con el funcionamiento de la "Fundación Argentina de Nanotecnología". Firmantes: Fiad, Mario y Giubergia, Miguel. Publicado en: Trámite Parlamentario $\mathrm{N}^{\circ}$ 58. Fecha: 18/05/2010.

Argentina. Proyecto de Ley Número de Expediente 2092-S-14. Marco para el Desarrollo y la Aplicación de la Nanotecnología, la Física Cuántica y la Biología Sintética. Autores: Negre De Alonso, Liliana, Rodríguez Saá, Adolfo. Publicado en: Diario de Asuntos Entrados $N^{\circ}$ 103. Fecha: 02/07/2014. 


\section{Documentos utilizados}

Argentina. Auditoría General de la Nación (2010). Resolución 032/2010. Auditoría de gestión por el período comprendido entre el 31/08/2005 y el 30/06/2008. Organismo auditado: Fundación Argentina de Nanotecnología (FAN) y Ministerio de Ciencia, Tecnología e Innovación Productiva (MCTeIP).

Argentina. Comité Nacional de Ética en la Ciencia y la Tecnología (2005). Declaración sobre el Decreto 380/05. Recuperado de http://www.mincyt. gob.ar/informes/declaracion-sobre-el-decreto-38005-11698

Argentina. MINCyT (2009). Nanotecnología. Boletín Estadístico Tecnológico del Ministerio de Ciencia, Tecnología e Innovación Productiva.

Argentina. MINCyT (2012a). Argentina Innovadora 2020. Plan Nacional de Ciencia, Tecnología e Innovación. Lineamientos Estratégicos 2012-2015. Buenos Aires, Ministerio de Ciencia, Tecnología e Innovación Productiva

Argentina. MINCyT (2012b). Empresas y Grupos de I+D de Nanotecnología de Argentina. Secretaria de Planeamiento y Políticas del Ministerio de Ciencia, Tecnología e Innovación Productiva.

Argentina. MINCyT (2013). Nanotecnología. Casos de asociatividad e innovación. Secretaría de Planeamiento y Políticas del Ministerio de Ciencia, Tecnología e Innovación Productiva.

Argentina. MINCyT (2016). Estudios de consultoría en el sector nanotecnológico. El futuro de las nanociencias y las nanotecnologías en Argentina. Estudio de prospectiva y vigilancia tecnológica. Dirección Nacional de Estudios, Subsecretaría de Estudios y Prospectiva de la Secretaría de Planeamiento y Políticas del Ministerio de Ciencia, Tecnología e Innovación Productiva.

Argentina. Ministerio de Educación, Ciencia y Tecnología (2006). Plan Estratégico Nacional de Ciencia, Tecnología e Innovación: "Bicentenario" (20062010). Secretaría de Ciencia, Tecnología e Innovación Productiva SECyT. 
Asociación Física Argentina (2005). Comunicado de la Comisión directiva con referencia al decreto 380/2005.

Giorgetti, J. (2001). Para Cambiar lo que Duele. Ideas. Proyectos. La política como herramienta de transformación. Buenos Aires. Recuperado de http://www.jorgegiorgetti.com.ar/publicaciones/libros/downloads/paracambiar-lo-que-duele-GIORGETTI.pdf

Observatorio Iberoamericano de la Ciencia, la Tecnología y la Sociedad (2011). La nanotecnología en Iberoamérica. Situación actual y tendencias. Documento de trabajo $\mathrm{n}^{\circ} 4$.

\section{Noticias citadas}

ANMAT (30 de Noviembre de 2012). Se conformó Grupo de Trabajo Interdisciplinario sobre Nanotecnología. Recuperado de http:/www.anmat.gov .ar/comunicados/Nanotecnologia.pdf

ANPCyT (29 de Septiembre de 2017). La Agencia reafirmó su apoyo a la innovación en nanotecnología. Recuperado de http:/www.agencia.mincyt .gob.ar/frontend/agencia/post/2739

CECTE. (s.f). Conferencia Internacional para la Investigación Responsable en Nanociencia y Nanotecnología. Recuperado de http:/www.cecte .gov.ar/conferencias-seminarios-y-talleres/\#conferencia-internacionalpara-la-investigacion-responsable-en-nanociencia-y-nanotecnologia

CECTE. (s.f). Primeras Jornadas "Ética en el Acceso al Conocimiento". Recuperado de http://www.cecte.gov.ar/conferencias-seminarios-y-talleres/\#primeras-jornadas-\%C3\%89tica-en-el-acceso-al-conocimiento

Ciencia Argentina en la Vidriera (3 de Octubre de 2013). Salvarezza habló del aumento de profesionales argentinos en nanotecnología. Programa 464. Recuperado de http://www.cienciaenlavidriera.com.ar/2013/10/03/programa-464-salvarezza-hablo-del-aumento-de-profesionales-argentinosen-nanotecnologia/ 
Ferrari, A. (25 de Septiembre de 2005). La batalla naval de los científicos argentinos. Página 12. Recuperado de https:/www.pagina12.com.ar/diario/elpais/1-56973-2005-09-25.html

Ferrari, A. (29 de Septiembre de 2005). Comité de Ética para la ciencia argentina pagada por la US Navy. Recuperado de https://www.pagina12 .com.ar/diario/sociedad/3-57141-2005-09-29.html

Ferrari, A. (02 de Noviembre de 2005). Dime quién te financia... Página 12. Recuperado de https://www.pagina12.com.ar/diario/sociedad/3-587092005-11-02.html

Foladori, G. (10 de Noviembre de 2006). La influencia militar estadounidense en la investigación de las nanotecnologías en América Latina. Col·lectiu Antimilitarista de Sant Cugat. Recuperado de https://www.nodo50. org/casc/spip.php?article180

Giorgetti, J. (03 de Julio de 2007). No sólo de soja vive Argentina. La Capital. Recuperado de http://www.jorgegiorgetti.com.ar/publicaciones/articulos/no-solo-soja-argentina.htm

Giorgetti, J. (22 de Agosto de 2006). Ciencia: apurarnos es un deber político. La Capital. Recuperado de http://www.jorgegiorgetti.com.ar/publicacion es/articulos/ciencia.htm

Iprofesional (2 de Agosto de 2006). El Gobierno apoyará iniciativas de nanotecnología. Recuperado de http://www.iprofesional.com/notas/31191El-Gobierno-apoyara-iniciativas-de-nanotecnologia?page_y $=0$

La Razón (2 de agosto de 2006). Nanotecnología: habrá financiamiento oficial. Recuperado de http://edant.larazon.com.ar/diario_lr/2006/08/02/21245000.htm

Luna, N. (16 de Abril de 2015). "Debemos dominar la tecnología para ser compradores inteligentes". Agencia TSS. Recuperado de http://www. unsam.edu.ar/tss/debemos-dominar-la-tecnologia-para-ser-compradoresinteligentes/ 
MAyDS (7 de Junio de 2017). Nanotecnología en la semana del Ambiente. Recuperado de https://www.toxicologia.org.ar/wp-content/uploads/2017/ 05/Programa-de-Nanotecnolog\%C3\%ADa-7-jun.pdf

Mi Club Tecnológico (18 de Diciembre de 2015). "Más que nanoproductos a la Argentina le conviene producir nanointermediarios". Recuperado de http:/www.miclubtecnologico.com.ar/blog/mas-que-nanoproductos-a-laargentina-le-conviene-producir-nanointermediarios/

MINCyT (11 de Septiembre de 2013). Se presentó la iniciativa Antena Tecnológica. Recuperado de http://www.mincyt.gob.ar/noticias/se-presentola-iniciativa-antena-tecnologica-9255

MINCyT (17 de Septiembre de 2008). Avanza la creación de un código de ética para la investigación en nanotecnología. Recuperado de http://www. mincyt.gob.ar/noticias/avanza-la-creacion-de-un-codigo-de-etica-para-lainvestigacion-en-nanotecnologia-4206

MINCyT (25 de Abril de 2013). "Manolito", el nuevo nanosatélite argentino ya está en órbita. Recuperado de http://www.mincyt.gob.ar/noticias/manolito-el-nuevo-nanosatelite-argentino-ya-esta-en-orbita-9553

MINCyT (25 de Abril de 2013). Argentina pone en órbita al "Capitán Beto", el primer nano satélite de plataforma abierta. Recuperado de http://www .mincyt.gob.ar/noticias/argentina-pone-en-orbita-al-capitan-beto-el-primer-nano-satelite-de-plataforma-abierta-4805

Página 12 (25 de Septiembre de 2005). "No habría que permitirlo". Entrevista a Gregorio Klimovsky. Recuperado de https://www.pagina12.com.ar/diario/elpais/subnotas/56973-18935-2005-09-25.html

Página 12 (25 de Septiembre de 2005). "La destrucción de la ciencia”. Entrevista a Mischa Cotlar. Recuperado de https://www.pagina12.com.ar/diario/elpais/subnotas/56973-18936-2005-09-25.html 
Página 12 (25 de Septiembre de 2005). "Tiene que haber control". Entrevista a Pablo Jacovkis. Recuperado de https:/www.pagina12.com.ar/diario/elpais/subnotas/56973-18937-2005-09-25.html

Página 12 (9 de Diciembre de 2005). “Actúan mercenariamente en pos de financiación, a cualquier costo". Recuperado de https://www.pagina12. com.ar/diario/sociedad/3-60247-2005-12-09.html

Premici, S. (21 de Enero de 2007). El impulso de la nanotecnología, patentes y estrategia de desarrollo. El nuevo nano orden económico. Página 12. Recuperado de https://www.pagina12.com.ar/diario/suplementos/cash/172806-2007-01-22.html

\section{Notas}

${ }^{1}$ Argentina. Proyecto de Ley Número de Expediente 264-S-01. Proyecto de ley sobre elaboración del plan estratégico de ciencia y tecnología. Autor: Moreau, Leopoldo Raúl Guido. Origen: Senado de la Nación. Publicado en: DAE 017/01. Fecha: 03/04/2001.

${ }^{2}$ El documento de trabajo también reservaba un apartado para la coordinación interjurisdiccional de las citadas actividades, en cabeza de la Jefatura de Gabinete de Ministros. Se ordenaba expresamente la participación de organismos tales como el Ministerio de Salud y acción Social, el Instituto Nacional del Agua y del Ambiente y la Secretaría de Recursos Naturales y Desarrollo Sustentable (entre muchos otros). Finalmente, el proyecto ponía en cabeza del Estado Nacional la obligación de realizar anualmente "un informe detallado del estado de la ciencia y la técnica en el territorio nacional, tanto en el sector público como en el privado".

${ }^{3}$ En ese momento, la Secretaría dependía del por entonces Ministerio de Educación, y estaba a cargo de promocionar y financiar las actividades de I+D en el país.

${ }^{4}$ A raíz del PAV, la ANPCyT financió cuatro redes de N\&N que cubrían las siguientes temáticas: materiales nanoestructurados y nanosistemas; sistemas moleculares, supramoleculares e interfaces; MEMs: diseño, simulación y fa- 
bricación; y bionanoestructuras. Estas redes estuvieron conformadas por al menos tres nodos de grupos o centros de investigación (MINCyT, 2012b), comenzaron a trabajar en 2006, fueron un aporte importante en la formación de la masa crítica en esta actividad y crearon las condiciones de trabajo para el avance de las N\&N (Salvarezza, 2011).

${ }^{5}$ Andrini y Figueroa (2008) reseñan que en 2004 la SeCyT, conjuntamente con representantes de la Unión Europea, organizaron en Buenos Aires una Reunión destinada a promover las capacidades nacionales en el área de las $N \& N$, a través de programas de investigación y redes Europeas.

${ }^{6}$ Argentina. Proyecto de Ley Número de Expediente 1357-D-05. Creación del Instituto Argentino de Nanotecnología, en el ámbito del Ministerio de Economía. Autor: Giorgetti, Jorge. Publicado en: Trámite Parlamentario ${ }^{\circ} 21$. Fecha: 31/03/2005.

${ }^{7}$ El proyecto propuso como objetivo el diseño de un programa para incentivar en la Argentina el desarrollo de la nanotecnología y la formalización de convenios con institutos internacionales. Se incluía dentro del instituto el funcionamiento de un Proyecto de Incubadoras de Productos Nanotecnológicos.

${ }^{8}$ Para entender mejor este modo de inscripción de los nano-objetos en los discursos parlamentarios, es necesario complementarlo con otras fuentes. En un documento de su autoría titulado "Para Cambiar lo que Duele. Ideas. Proyectos. La política como herramienta de transformación", Giorgetti sostiene que la revolución tecnológica es la nueva fórmula de la creación de la riqueza (p. 26), y que la tecnología que impulsa la globalización debe ser utilizada para "ayudar a los pobres con una clara y decidida acción política" (p. 21). El legislador entendía que el punto de partida para este desarrollo era el "negocio de la cadena agroalimentaria" (p. 26). Esto sería ampliado por Giorgetti en algunas notas de opinión escritas en el Diario La Capital. En una de ellas, por ejemplo el diputado expresaba que "debemos entender que la biotecnología en general y la nanotecnología aparejan nuevas formas de hacer negocios" (Giorgetti, 2006).

${ }^{9} \mathrm{Al}$ poco tiempo de presentado el proyecto, el legislador fue invitado a un Seminario de Biotecnología programado por el Departamento de Estado de 
EE.UU., el Departamento de Agricultura, (USDA), la Administración de Alimentos y Drogas (FDA) y la Agencia de Protección del Medio Ambiente (EPA). El seminario tuvo lugar en Washington D.C., del 9 al 16 de Julio de 2005, e incluyó temas de biotecnología agrícola (siembra, percepción ciudadana de los riesgos, etc.).

${ }^{10}$ Algunas fuentes aclaran que la iniciativa, antes de que la tomara el Ministerio de Economía a propuesta del Balseiro, se gestó en el Instituto, que tenía una estrecha relación de colaboración con Lucent que databa de casi veinte años. Argentina. Proyecto de Resolución Número de Expediente 2617-D-2010. Pedido de informes al poder ejecutivo sobre diversas cuestiones relacionadas con el funcionamiento de la "Fundación Argentina de Nanotecnología", dependiente del Ministerio de Ciencia y Tecnología e Innovación Productiva. Publicado en: Trámite Parlamentario $N^{\circ}$ 43. Fecha: 28/04/2010.

${ }^{11}$ En el Acta de Constitución, se definió a la FAN como una "entidad civil sin fines de lucro, bajo la forma jurídica de Fundación", y se fijó su domicilio en la Ciudad Autónoma de Buenos Aires. El Estado nacional aportó como capital inicial la suma de doce mil pesos y se obligó a integrar diez millones de dólares en los cinco años subsiguientes.

${ }^{12}$ En el Estatuto, se previó un Consejo de Administración como órgano de dirección y administración, con un mínimo de seis miembros y un máximo de nueve. También se incluyó un órgano unipersonal denominado "Director Ejecutivo" para las funciones operativas y un Consejo Asesor como órgano colegiado con funciones consultivas (con un mínimo de cinco miembros y un máximo de veinte). El Estado Nacional se reservó facultades en carácter de miembro fundador, como la reforma estatutaria, la facultad de designar a los integrantes del Consejo de Administración, la posible fusión con entidades similares y la disolución. Nota aclaratoria: Por art. $1^{\circ}$ de la Resolución $\mathrm{N}^{\circ}$ 579/2005 del Ministerio de Economía y Producción se amplió a nueve (9) la cantidad de miembros del Consejo de Administración. Mediante la Ley 26.338 de 2007 la FAN pasó a la órbita del Ministerio de Ciencia, Tecnología e Innovación Productiva.

${ }^{13}$ De los fundamentos del Decreto también surge que el desarrollo del sector debe ser impulsado y compartido tanto por el Sector Público como por el Sec- 
tor Privado, y debe estar orientado a "un continuo incremento de la productividad, contribuyendo a través de ello a la reducción de la pobreza y de las disparidades que hoy existen en la sociedad argentina".

${ }^{14}$ Este modelo de N\&N, asentado en el discurso de la innovación y la competitividad y en los modelos globales de política científica y tecnológica -v.g. la iniciativa estadounidense NNI (National Nanotechnology Initiative) de 2000-, ha motivado críticas en los ESCT locales (cfr. Vila Seoane, 2014; Invernizzi et al, 2014; Foladori, 2016; Hurtado et al, 2017).

${ }^{15}$ El diario Página 12 reproduce otra opinión de Barañao, en la que el Director de la ANPCyT expresaba que "La única manera de actuar inteligentemente es a través de la generación de patentes propias que no interfieran con las patentes extranjeras. Hay que aprovechar este tiempo inicial para realizar las innovaciones que más le convengan al país y negociar a futuro con otras naciones. Es decir, poder revertir la situación donde una empresa copa el mercado o controla una tecnología clave, como en el caso de las semillas de Monsanto" (Premici, 2007)

${ }^{16}$ Dos argumentos sostienen este considerando: que este proceder no es compatible con las necesarias transparencia y seriedad que deben avalar una iniciativa y que "la Argentina cuenta con investigadores en estos temas cuyo criterio y experiencia deben ser aprovechados al momento de buscar y decidir cuál o cuáles van a ser los nichos que más convienen a su desarrollo".

${ }^{17}$ Argentina. Proyecto de Resolución Número de Expediente 2844-D-2005. Pedido de informes al Poder Ejecutivo sobre los objetivos de las políticas científicas y la creación de la Fundación Argentina de la Nanotecnología (Resolución del ME NS 380/05). Publicado en: Trámite Parlamentario $\mathrm{N}^{\circ} 52$. Fecha: 13/05/2005.

${ }^{18}$ Para Puig de Stubrin, la ley $\mathrm{N}^{\circ} 25.467$, que crea la Agencia Nacional de Promoción Científica y Tecnológica, establece claros mecanismos de concursos y licitaciones, y la ley 24.156 de administración financiera reclama dictámenes previos cuando el Estado aporta fondos a una Fundación en la que participa el sector privado. 
${ }^{19}$ El CECTE fue creado en abril de 2001 mediante la Resolución 004/2001 de la Secretaría de Ciencia, Tecnología e Innovación Productiva y desde 2007 funciona en el ámbito del Ministerio de Ciencia, Tecnología e Innovación Productiva.

${ }^{20}$ Del análisis normativo el CECTE concluyó que "un decreto que destine importantes sumas de dinero a una Fundación creada sin obligación de adhesión al sistema legal resultaría contrastante con la estructura tan cuidadosamente diseñada por el Congreso de la Nación Argentina, en manifiesto perjuicio al principio de igualdad, de tan alto valor ético y jurídico" y recomienda tanto para el caso estudiado como para casos futuros que "se observen los lineamientos de la Ley 25.467 a fin de garantizar que las decisiones progresen sobre bases éticas estrictas".

${ }^{21}$ La controversia que despertaron estos contratos de colaboración y la posible aplicación militar de los desarrollos motivó incluso la crítica del epistemólogo Gregorio Klimovsky (Página 12, 2005a), del matemático Mischa Cotlar (Página 12, 2005b) y del -por entonces- decano de la Facultad de Ciencias Exactas de la Universidad de Buenos Aires (Página 12, 2005c).

${ }^{22}$ Argentina. Proyecto de Resolución Número de Expediente 5416-D-2005. Pedido de Informes al Poder Ejecutivo sobre el Financiamiento de Proyectos de Investigación Científica, Desarrollo Tecnológico o Innovación Productiva. Autores: Puig de Stubrin, Lilia; Storani, Federico; Negri, Mario. Publicado en: Trámite Parlamentario no 132. Fecha: 16/09/2005.

${ }^{23}$ Argentina. Proyecto de Resolución Número de Expediente 0753-D-2006. Pedido de Informes al Poder Ejecutivo sobre el Financiamiento de la Conferencia de Carácter Científico Realizada en el Hotel Tunquelen, Bariloche, Provincia de Rio Negro, por parte de la Oficina de Investigaciones Navales y de la Fuerza Aérea de Estados Unidos de América. Autores: Rodríguez, Marcela; García, Susana; Bisutti, Delia. Publicado en: Trámite Parlamentario n ${ }^{\circ}$ 13. Fecha: 17/03/2006.

${ }^{24} \mathrm{El}$ diario reproduce textualmente la opinión del especialista (Daniel Grain): "El camino abierto en el terreno de la biotecnología en la Argentina muestra la senda que seguirán estos desarrollos: vía libre para las empresas, ningún 
control sobre los impactos ambientales y ausencia total de debate público" (Premici, 2007).

${ }^{25}$ Lupi amplía su razonamiento de la siguiente manera: "Durante la etapa neoliberal de la Argentina, la política indicaba que sólo podíamos incorporar tecnología desarrollada en el exterior, por lo tanto no se necesitaba ninguna política industrial. Hoy, la política nos estimula a jugar un papel más relevante, para generar productos que se comercialicen en el mercado" (Premici, 2007).

${ }^{26}$ A raíz de ello, se presentaron dos proyectos legislativos de pedido de informes al Poder Ejecutivo Nacional. El primero, propiciado por un bloque de siete diputados de la UCR: Argentina. Proyecto de Resolución Número de Expediente 2617-D-2010. Pedido de informes al poder ejecutivo sobre diversas cuestiones relacionadas con el funcionamiento de la "Fundación Argentina de Nanotecnología", dependiente del Ministerio de Ciencia y Tecnología e Innovación Productiva. Publicado en: Trámite Parlamentario $\mathrm{N}^{\circ}$ 43. Fecha: 28/04/2010. El segundo, de los diputados Mario Raymundo Fiad (UCR) y Miguel Ángel Giubergia (UCR). Proyecto de Resolución, Expediente Diputados: 3353-D-2010. Pedido de informes al Poder Ejecutivo sobre diversas cuestiones relacionadas con el funcionamiento de la "Fundación Argentina de Nanotecnología". Publicado en: Trámite Parlamentario $N^{\circ}$ 58. Fecha: $18 / 05 / 2010$.

${ }^{27}$ Argentina. Proyecto de Ley Número de Expediente 3279-D-2005. Plan Nacional Estratégico de Desarrollo de Micro y Nanotecnologías, y derogación del Decreto 380/05. Publicado en: Trámite Parlamentario $n^{0}$ 65. Fecha: 02/06/2005.

${ }^{28}$ Este fondo se planteaba para financiar actividades y proyectos de investigación aplicada, desarrollo tecnológico, transferencia tecnológica e innovación productiva y formación de recursos humanos en la materia. Con esto se buscaba evitar el conflicto de intereses que surge al reunir en una misma institución las acciones de promoción y ejecución (como lo hace la actual FAN).

${ }^{29}$ Para este grupo de diputados se debía tener presente que "las tecnologías para el desarrollo de aplicaciones de la nanotecnología son bastante distintas de las aplicaciones de uso agrícola. Los costos de la infraestructura son muy 
elevados. Debería existir una decisión política de muy largo plazo en qué áreas de la nanotecnología debemos concentrar nuestros esfuerzos, ya que no estamos en condiciones de realizar inversiones de miles de millones de dólares como se hacen en los países desarrollados."

${ }^{30}$ Argentina. Proyecto de Ley Número de Expediente 7277-D-2006. Ley Marco de la Industria Nanotecnológica. Publicado en: Trámite Parlamentario $\mathrm{n}^{\circ}$ 188. Fecha: 07/12/2006.

${ }^{31}$ El propio diputado Giorgetti en sus artículos periodísticos comienza a advertir las contradicciones del modelo de monocultivo sojero argentino (2007). Se comienza a percibir que en esta etapa el discurso de la biotecnología agrícola dejaba de abrigar al discurso de las $\mathrm{N} \& \mathrm{~N}$.

${ }^{32}$ Argentina. Proyecto de Ley Número de Expediente 4853-D-2008. Ley marco de la industria nanotecnológica (INNATEC): objetivo, definiciones, autoridades. Publicado en: Trámite Parlamentario N 114. Fecha: 04/09/2008

${ }^{33}$ Mediante la Ley 26.338 de 2007, modificatoria de la Ley de Ministerios (t.o Decreto 438/92), la FAN pasó a la órbita del Ministerio de Ciencia, Tecnología e Innovación Productiva. La FAN, al año siguiente realizó una convocatoria donde se anunciaba que se habían presentado veinte empresas "que tienen proyectos avanzados o productos con una base de nanotecnología". Hubert (2014) aclara que la FAN financió, a través de su primera convocatoria, 9 ideas-proyecto con orientación tecnológica e industrial y que, mientras que las redes formaban parte de la investigación pública, los proyectos de la FAN estaban directamente orientados hacia los actores privados.

${ }^{34}$ En el Plan, la nanotecnología es un área considerada como la de mayor potencialidad dentro del nuevo paradigma tecnológico, por lo que ofrece una ventana de oportunidad para países en vías de desarrollo como la Argentina, en la medida en que los cambios en la estructura productiva mundial abren un espacio para los "nuevos jugadores". Al respecto, esta tecnología sobresale por el camino relativamente corto entre la innovación y la producción y por la posibilidad de patentamiento y disminución de la dependencia tecnológica (MINCyT, 2012a, p. 60). 
${ }^{35}$ En resumen, pueden encontrarse Proyectos de Investigación y Desarrollo (PID) correspondiente al Fondo para la Investigación Científica y Tecnológica (FONCYT); Empresas de Base Tecnológica (Empretecno) y Fondos Sectoriales Nano (FS) del Fondo Argentino Sectorial (FONARSEC); ANR y Emprendedores FONSOFT del Fondo Fiduciario de Promoción de la Industria del Software (FONSOFT); ANR TEC, Artículo N², Asistencias Tecnológicas (ASIS-TEC) y Recursos Humanos Altamente Calificados (RRHH AC) del FONTAR (ANPCyT, 2017).

${ }^{36}$ Hubert (2014) explica que tras la creación de los Fondos Sectoriales la actividad de la FAN se reorientó hacia la organización de reuniones de intercambio de información. En particular, los encuentros Nano Mercosur que agruparon a actores públicos y privados de las N\&N. Lupi, como presidente de la FAN, ha expresado que la misión de la Fundación "estaría cumplida si logramos dejar algunos casos paradigmáticos que demuestren que la nanotecnología es negocio o que es conveniente en algún aspecto que beneficie a la sociedad argentina" (Luna, 2015).

${ }^{37}$ Argentina. Proyecto de Ley Número de Expediente 2092-S-14. Marco para el Desarrollo y la Aplicación de la Nanotecnología, la Física Cuántica y la Biología Sintética. Publicado en: Diario de Asuntos Entrados $N^{\circ} 103$. Fecha: 02/07/2014.

${ }^{38}$ Este proyecto parlamentario expresaba que la propuesta "es hecha con la idea de que estos fundamentales avances de la humanidad respeten en todo momento la vida" y advirtiendo que "estas nuevas tecnologías pueden conllevar a riesgos y peligros si son mal utilizadas". También expresaba que las posibilidades de esos campos del conocimiento deben estar "totalmente al servicio de todas las personas y de todos los seres que habitan nuestro Planeta". No obstante ello, el texto no preveía en su articulado mecanismos específicos para asegurar la observancia de los principios que buscaba tutelar.

${ }^{39}$ La norma reglamenta el Decreto 987/2006, que aprobó el modelo de Contrato de Préstamo No 1728/OC-AR con destino al Programa de Modernización Tecnológica III. 
${ }^{40}$ La Unidad de Gestión Socio Ambiental (UGSA) presta apoyo técnico, asistencia y colaboración en temas de gestión ambiental e higiene y seguridad en el trabajo, a la Presidencia de la Agencia Nacional de Promoción Científica y Tecnológica (ANPCyT) y a los diferentes fondos que se encuentran bajo su órbita, a saber: FONTAR, FONCyT y FONARSEC. Asimismo colabora con aquellas áreas que requieran su intervención a través de los diferentes programas de financiamiento externo.

${ }^{41}$ El encuentro fue organizado por la Dirección de Relaciones Internacionales del Ministerio de Ciencia, Tecnología e Innovación Productiva; el Comité Nacional de Ética en la Ciencia y la Tecnología (CECTE), la Fundación Argentina de Nanotecnología (FAN), el Centro Argentino-Brasileño de Nanociencia y Nanotecnología (CABNN), el Programa Argentino-Brasileño de Ética en la Ciencia y la Tecnología (PABECyT), y recibió el auspicio de la Dirección General de Investigación de la Comisión Europea (MINCyT, 2008)

${ }^{42}$ En este encuentro Peteris Zigalvis, responsable de la Unidad de Gobernanza y Ética de la Dirección General de Investigación de la Comisión Europea, presentó el Código de Conducta para la Investigación Responsable en Nanociencia y Nanotecnología adoptado en 2008 por la Comunidad Europea. Según el MINCyT en este encuentro Zigalvis expresó que "la cuestión principal respecto de los riesgos, es el tema de la seguridad de las nano partículas. Y ante la situación actual, una salida para afrontar este riesgo es el principio de precaución y la implementación de un código de conducta" (MINCyT, 2008).

${ }^{43}$ Entre las normas publicadas por el Comité ISO/TC 229, se puede destacar la serie ISO/ TS 80004, que se ha encargado de fijar estándares en el vocabulario, sobre términos básicos (80004-1:2015), nano-objetos (80004-2:2015), nano-objetos de carbono (80004-3:2010), materiales nanoestructurados (80004-4:2011), interfaz nano/bio (80004-5:2011), caracterización del nanoobjeto (80004-6:2013), diagnóstico y terapéutica en salud (80004-7:2011), procesos de nanomanufacturado (80004-8:2013) y fenómenos cuánticos en nanotecnología (80004-12:2016). Otras normas de vocabulario para esta serie actualmente se encuentran en fase de estudio y elaboración. 
${ }^{44}$ ISO/TC 229/CAG: Presidente del Grupo Asesor. ISO/TC 229/JWG 1: Grupo dedicado a cuestiones terminológicas y de nomenclatura. ISO/TC 229/JWG 2: Grupo dedicado a medidas y caracterizaciones. ISO/TC 229/TG 2: Sobre consumo y dimensiones sociales de las nanotecnologías. ISO/TC 229/TG 3: Sobre nanotecnologías y sostenibilidad. ISO/TC 229/WG 3: Dedicado a salud, seguridad y aspectos ambientales de las nanotecnologías. ISO/TC 229/WG 4: Abocado al estudio de las especificaciones materiales. ISO/TC 229/WG 5: Estudia productos y aplicaciones.

${ }^{45} \mathrm{CGN}$ : Indica que se trata de un documento cuyo estudio se ha completado, restando tan sólo su análisis por el Comité General de Normas, en los aspectos formales de coordinación y compatibilización con otras normas

${ }^{46}$ ANT: Significa que en el organismo de estudio se están reuniendo antecedentes sobre ese tema, o bien que el primer documento preparado lleva ese nombre por estar incompleto o porque se supone que será pasible de muchas modificaciones.

${ }^{47}$ Según Fiorani (2011, p. 12) "luego del entusiasmo inicial por las oportunidades -aparentemente ilimitadas- de sus aplicaciones, la comunidad nanotecnológica tomó una postura conservadora para contemplar las posibles implicancias en la salud y la seguridad de las personas y el ambiente expuestos a nanomateriales".

${ }^{48}$ El Comité ISO/TC 229 también ha avanzado en normas estandarizadas en materia de evaluación de riesgos, como la norma ISO/TR 13121:2011, de evaluación de riesgo de nanomateriales, ISO/TR 13329:2012, de preparación de fichas de datos de seguridad para nanomateriales y la norma ISO/TS 12805:2011, que orienta sobre especificaciones materiales de nano-objetos. De forma concordante, el Comité también ha publicado estándares para gestionar los riesgos de estas tecnologías en entornos laborales, como la norma ISO/TR 12885:2008, sobre prácticas de salud y seguridad en entornos laborales relevantes para las nanotecnologías, o las normas ISO/TS 12901-1:2012 y 129012:2014, de gestión de riesgos laborales aplicado a nanomateriales de ingeniería. Otra norma de relevancia es la ISO/TS 13830:2013, sobre etiquetado voluntario para productos de consumo que contengan nano-objetos manufacturados. 
${ }^{49}$ La peculiaridad del régimen francés es que la expresión substances à l'état nanoparticulaire es una categoría "anómala" que no reconoce antecedentes en ningún lugar del mundo y sólo se explica apelando a las particularidades que revistió la controversia en ese país (cfr. Laurent, 2017, p. 117).

${ }^{50} \mathrm{El}$ documento destaca que "la baja mención de los grupos de I+D sobre esta temática sugiere que este no es una cuestión prioritaria al menos por el momento. De todos modos, cabe destacar que a nivel internacional son fuertes los debates y las investigaciones sobre los posibles impactos de la Nanotecnología en la sociedad, principalmente debido a los efectos tóxicos de algunos materiales en la nanoescala" (2012b, p. 32).

${ }^{51}$ Así, "para algunos de los encuestados la falta de regulaciones o su escaso desarrollo genera incertidumbre a la hora de tomar la decisión de desarrollar o no un producto. Esto sucede, en particular, en las áreas relacionadas con aplicaciones en salud humana, ya que tal vez no sea fácil introducir los desarrollos en el mercado, como se esperaba inicialmente" (MINCyT, 2012b, p. 32).

${ }^{52}$ Estudios de consultoría en el sector nanotecnológico. El futuro de las nanociencias y las nanotecnologías en argentina. Estudio de prospectiva y vigilancia tecnológica. Préstamo BIRF N $7599 / A R$ - Licitación No 05/09. El Consorcio estuvo integrado por el Observatorio Tecnológico (OTEC) del Departamento de Ingeniería Industrial de la Facultad de Ingeniería de la Universidad Nacional de Mar del Plata (Argentina), el Instituto de Bioingeniería de Cataluña (IBEC) y la Fundació Hospital Universitari Vall d'Hebron- Institut de Recerca (VHIR) (España).

${ }^{53} \mathrm{El}$ estudio entiende que el tema ha permanecido relegado, y que la necesidad de enfrentarlo se debe a la probabilidad de que en el mediano plazo Argentina sea un país exportador de nanomateriales (2016, p. 72). Como grandes estrategias para alcanzar los objetivos, la consultoría estima necesario facilitar la inversión extranjera en el país, agilizar los trámites de importación y diseñar un marco normativo adecuado.

${ }^{54}$ Resaltando que cuando se trata de productos con nanomateriales, la legislación y las normativas deben estar orientadas al control de productos alimentarios, médicos y farmacéuticos, de modo de garantizar la seguridad de 
la población (2016, p. 72).

${ }^{55}$ Ampliando este punto, el texto expresa que "para poder realizar esta evaluación de un modo fiable, dado que el tamaño, la forma y otras propiedades fisicoquímicas de una nanopartícula pueden dar lugar a cambios en los efectos producidos, es necesario especificar las características de cada producto de nanopartículas; el concepto de dosis de exposición tiene que ser definido en términos de número de partículas y/o área total y no únicamente en términos de masa como convencionalmente" (2016, p. 193).

${ }^{56}$ Según el estudio, esta plataforma permite gestionar, ordenar, clasificar y actualizar de forma integrada distintas fuentes de información, es personalizable en función de las necesidades y requerimientos de cada empresa u organización y es útil para organizaciones y usuarios finales interesados en los contenidos, y en recibir notificaciones frecuentes de novedades (p. 166).

${ }^{57}$ Según el comunicado de la ANMAT, de fecha 30 de Noviembre de 2012, el grupo se abocó a realizar un diagnóstico de situación sobre aspectos científicos regulatorios, en base a documentos nacionales e internacionales, y a sistematizar información específica sobre el tema. El comunicado preveía una segunda instancia en la que se convocarían referentes de organismos técnicos y de distintas instituciones académicas, a fin de iniciar una etapa de intercambio y actualización recíproca continua.

${ }^{58}$ Los ESCT locales han puesto de manifiesto la diversidad de trayectorias al interior de las redes de investigación, la falta de consenso respecto de los contenidos de las N\&N (rupturas y continuidades) y las distintas caracterizaciones y reajustes que las redes inscriben en los nano-objetos (cfr. Hubert y Spivak L'Hoste, 2009).

${ }^{59}$ Para Lupi, el principal problema que tenía la nanotecnología es "que se conocía muy bien dentro de los ámbitos científicos, muy poco dentro del ámbito industrial y nada en la sociedad" (Mi Club Tecnológico, 2015). En este sentido, debe tenerse en cuenta al Grupo de Nanotecnología del Instituto Nacional de Tecnología Industrial (INTI), que en los '80 desarrolló un microscopio de efecto túnel por el cual se lograron las primeras imágenes de resolución atómica en Latinoamérica. Se destaca también la creación en 1999 del Labo- 
ratorio de Nanoscopías. Por último, cabe destacar los sucesivos Encuentros de Superficies y Materiales Nanoestructurados, una iniciativa nacida en 2001 en el seno de la Comisión de Energía Atómica en Bariloche, que acostumbra convocar todos los años investigadores de N\&N de toda la región.

${ }^{60}$ Para Lupi, es el Estado quien debe identificar las oportunidades de la nanotecnología para nuestra sociedad, formar recursos humanos en el área, incentivar redes de colaboración, crear una masa crítica de expertos y luego promover la creación de plataformas de vinculación entre empresas e investigadores (2012, p. 47). En algunas áreas considera que debe darse lugar a un "demandante privilegiado" que financie por fuera de las reglas generales del mercado (2012, p. 46). 